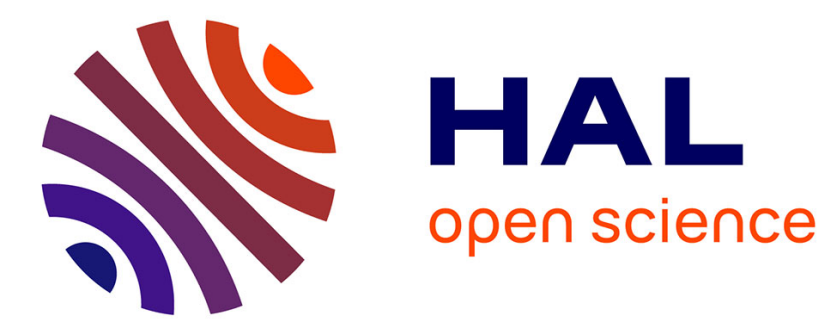

\title{
An approach for predicting the internal behaviour of ball bearings under high moment load
}

Christophe Bovet, Laurent Zamponi

\section{To cite this version:}

Christophe Bovet, Laurent Zamponi. An approach for predicting the internal behaviour of ball bearings under high moment load. 2015. hal-01154703

\section{HAL Id: hal-01154703 \\ https://hal.science/hal-01154703}

Preprint submitted on 27 May 2015

HAL is a multi-disciplinary open access archive for the deposit and dissemination of scientific research documents, whether they are published or not. The documents may come from teaching and research institutions in France or abroad, or from public or private research centers.
L'archive ouverte pluridisciplinaire HAL, est destinée au dépôt et à la diffusion de documents scientifiques de niveau recherche, publiés ou non, émanant des établissements d'enseignement et de recherche français ou étrangers, des laboratoires publics ou privés. 


\title{
An approach for predicting the internal behaviour of ball bearings under high moment load
}

\author{
Christophe Bovet ${ }^{\mathrm{a}, \mathrm{b}, *}$, Laurent Zamponi ${ }^{\mathrm{b}}$ \\ ${ }^{a}$ Aix-Marseille Université, CNRS, ISM UMR 7287, 13288, Marseille cedex 09, France \\ ${ }^{b}$ Airbus Helicopters, Aéroport International Marseille Provence, 13725 Marignane, France
}

\begin{abstract}
This paper presents a modelling approach for predicting the internal dynamic behaviour of ball bearings under high moment loads. This type of loading is a specific feature of helicopter main gear boxes because of special design rules and the high structural flexibility of such systems. The ball bearing model proposed here is not limited to planar systems and incorporates several different phenomena such as contact deformation, elastohydrodynamic contact, internal clearance and cage run-out. The cage-race interaction is treated as a hybrid short journal model, which ensures continuity of the contact force at the transition from the hydrodynamic to metal-to-metal regime. In the dynamic analysis of such a severely loaded bearing, the dependence of the shaft-to-inner-ring force on inner race position cannot be neglected. An equivalent viscoelastic hinge joint has been developed, it produces an additional force that represents the overall rigidity of the system. The stiffness parameters of the joint are identified using global finite element simulations. A ball bearing loaded with two different moments is chosen as an example. Relevant results concerning the internal dynamic behaviour are given. The predicted cage trajectory has been compared to experimental observations, and good agreement has been found.
\end{abstract}

Keywords: Multibody dynamics, Bearing dynamics, Contact Analysis, Cage Analysis, Realistic Joint

\section{Introduction}

In the aircraft industry, and in particular the manufacture of helicopters, the quest for maximum performance requires the power weight ratio to be maximized. A power transmission gearbox of $300 \mathrm{~kg}$ can transmit several megawatts 5 from the engines to the rotor (see FIG 1). Weight saving is essentially achieved by reducing the number of parts and their thicknesses. Such a strategy leads to

\footnotetext{
* Corresponding author. Tel.:+3342939096

Email addresses: christophe.bovet@etu.univ-amu.fr (Christophe Bovet), christophe.bovet@airbus.com (Christophe Bovet), laurent.zamponi@airbus.com (Laurent Zamponi)
}

Preprint submitted to Mechanism and Machine Theory

July 23, 2014 
increasingly flexible housings and shafts. Associated with the high power transmitted, this increasing flexibility causes relatively large strains in the power transmission gearbox. Rolling bearings are naturally affected by housing deformation since any misalignment of rolling bearing races may cause damage to rolling element bearings and premature failure [1. This structural deformation leads to an additional moment load on the rolling bearings. Also, the ball separator or cage becomes a critical component. Previously there simply to ensure a uniform distribution of the rolling elements, it now experiences significant 15 stresses, and cage fracture due to material fatigue frequently occurs. In power transmission gearboxes, rotating shafts are usually supported by three rolling bearings; two roller bearings support radial loads whereas the ball bearing may experience mainly thrust loads. This load distribution is obtained on account of a large clearance between the outer race of the ball bearing and its housing.

20 As each rolling bearing must experience an appropriate load, improved system reliability is expected. Thus, the load experienced by ball bearings comprises a thrust load and an additional moment load inherent in structural deformation. This type of loading appears highly specific to the helicopter industry and is largely ignored in the literature. Indeed, the majority of rolling bearing dynamic analyses published in the literature only consider axial or radial loads.

Because of their critical role, rolling element bearings have been widely investigated. Initial studies focused on static and quasi-static analyses. Stribeck was among the first to investigate the load distribution in rolling elements. Later, Lundberg and Palmgren studied the fatigue behaviour of bearings. Perhaps the

30 first computer code to carry out static analyses of rolling bearings is credited to Jones [2]. This code was able to predict the load distribution, stiffness and fatigue life of rolling bearings. The work of Harris is also of great importance, with an excellent review of the work to be found in 3 . To address the problem of kinematic indeterminacy in quasi-static analyses, Jones 2 proposed the very commonly used race control hypothesis. The hypothesis states that the spin velocity of contacts between rolling elements and race is zero on the race that provides the larger friction torque. The hypothesis determines the angular velocity of the rolling elements. Quasi-static analyses are very useful in the design of rolling bearings since they provide a fairly realistic load distribution for the 40 rolling elements, equivalent stiffness and predictions of fatigue life. However, it suffers from several limitations. Indeed, any treatment of cage behaviour, skidding or skewing phenomena, and time varying loads are outside the realm of the model. These limitations drove the development of dynamic rolling bearing models.

45 Walters 4 initiated this approach, proposing a dynamic model which integrated the equation of motion of the cage in angular contact ball bearing. Kannel and Bupara submitted a simplified approach that makes use of the race control hypothesis [5] later contested by Meeks [6]. A major advance in the understanding of the dynamic behaviour of rolling bearings was made by

so Gupta with the well-known computer code ADORE [7]. The author developed a generalized dynamic formulation for various types of rolling bearings. The geometrical generalizations allowed geometrical imperfections to be modelled and 
manufacturing tolerances to be optimized [8. The race control hypothesis was replaced by the dynamic equilibrium of each rolling bearing component. How-

55 ever, ADORE seems unable to simulate ball bearings under high moment loads. In any event, no ball bearing dynamic analysis considering high moment loads has been published in the literature. The absence of radial loading coupled to the fact that the loaded race is floating leads to an unexpected behaviour of the inner race. This unexpected behaviour may be inherent in the too restricted ${ }_{60}$ scope of the Gupta's approach which neglects the link between the shaft and the inner race.

In the realm of vibration analysis, several models have been proposed to investigate the non-linear dynamic behaviour of a rotor-bearing system. These models often simplify physical phenomena such as lubricant traction or cage race 65 interaction. The aim of these studies is to underline the influence of specific defects on the vibrational response. Non-linearities due to internal localized defects [9] or internal clearances [10] were investigated. Harsha and Nataraj proposed an analytical model to show the effect of the run-out of the cage [11, 12. Due to run-out of the cage, the rolling elements no longer remain equally 70 spaced. Surface waviness [13, 14, ring flexibility [15, 16, and fluctuations in rotational speed [17] were also studied.

Dynamic modelling of multibody systems allows analysis, design, optimization and control of complex mechanical systems [18 [19. Joints were modelled in the past as idealized or simplified but the use of more realistic joints is of 75 growing interest with models of real cylindrical or spherical joint being developed 20 24]. Recently, a model of a planar deep groove ball bearing that can be used in planar multibody systems has been proposed [25]. This model model was used to estimate dynamic loads in a deep groove ball bearing [26, 27] and extended to include waviness defects $[28$. Though several physical simplifica80 tions were made, the multibody dynamic formalism appears to be well suited to the simulation of rolling bearings. Another interesting aspect of this approach is that the developed model can easily be included in a global dynamic study. Stacke et al. were the first to developed the bearing simulation tool BEAST based on a multibody dynamic formalism 29. The BEAST computer code allows the

85 dynamic internal behaviour of rolling bearings to be analysed with a detailed treatment of contacts [30.

This paper is concerned with the dynamic modelling of a ball bearing under high moment loads. The approach presented here draws on multibody dynamic formalism but also proposes a detailed treatment of internal interactions for rolling contact or cage behaviour. This approach has already been used in a previous work to predict ball bearing behaviour in the event of failure of the lubrication system [31. The proposed model is not limited to planar systems and it includes several phenomena such as internal clearance and cage runout. The contact force and moment calculations of rolling contacts are based

95 on the theory elastohydrodynamic lubrication [32, 33. Lubricant traction is approximated assuming isothermal conditions [34. The equivalent load to which the ball bearing is subject is highly specific to helicopter main gearboxes as these experience only axial loads and moment loads. In such cases, standard 
local approaches are unable to reproduce experimental observations because of the unexpected behaviour of the free race. Thus, the influence of the rest of the system can no longer be neglected. An equivalent viscoelastic hinge joint has been developed incorporating the overall stiffness of the helicopter main gearbox. Rigidity and damping parameters of the joint are identified via global finite element simulations. Moreover, the bearing excitation is so 105 severe that metal-to-metal contact occurs between the cage and the cage guiding race. To simulate this phenomenon and ensure continuity of the corresponding contact law, a hybrid short journal model has been developed. This considers the transition between the pure hydrodynamic and dry contact regimes. Flores et al. 20, 22 presented a similar approach for simulating journal bearings.

The paper is organized in the following manner. Section 2 presents the overall modelling approach and the equations of motion of a constrained multibody dynamic system are briefly reviewed in Section 3 . Section 4 describes the internal ball bearing interactions. Finally, two numerical examples and relevant results are given in section 5. Results are discussed and compared with experimental observations.

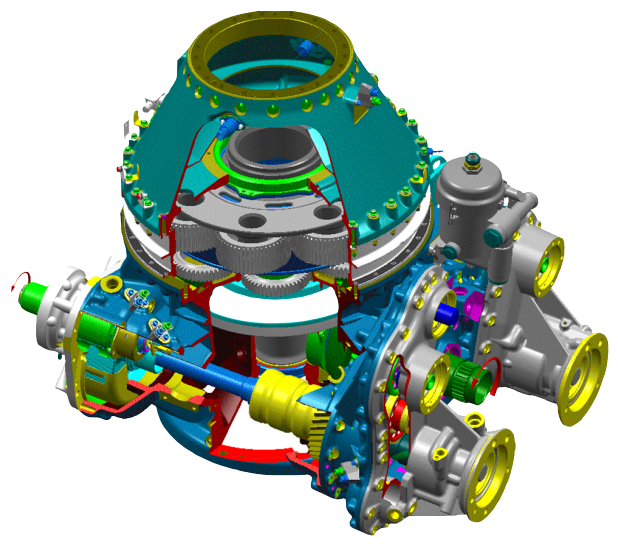

Figure 1: Helicopter main gearbox

\section{Modelling strategy of the system}

This section presents the strategy adopted for managing the dynamic analysis of rolling bearings of helicopter power transmission gearboxes. The dynamic analysis aims to predict cage instabilities and localized non-linear phenomena as ball skidding or impacts. Simplified models like such as those proposed in the realm of vibration analysis are not satisfactory. Such assumptions as race control or the regular distribution of rolling elements are excluded. Direct integration of the equations of motion of the complete gearbox, including the structural flexibility, gears and rolling bearings, represents a prohibitive cost in terms of ${ }_{125}$ CPU time and memory. Hence an alternative strategy with a restricted scope 
has been developed. The dynamic analysis is restricted to one rolling bearing at a time.

In the approach proposed by Gupta, the load is applied to the inner race which is free to move to balance the prescribed load and rolling element contacts. The outer race is rigidly connected to the housing. The main necessary item of data is the equivalent load seen by the rolling bearing. Assuming rigid shafts and housings, a simple static equilibrium provides an estimate of this equivalent load 3. However, this method is not suitable for aircraft gearboxes since it neglects the structural flexibility of the mechanism and it grossly underestimates the value of the equivalent load.

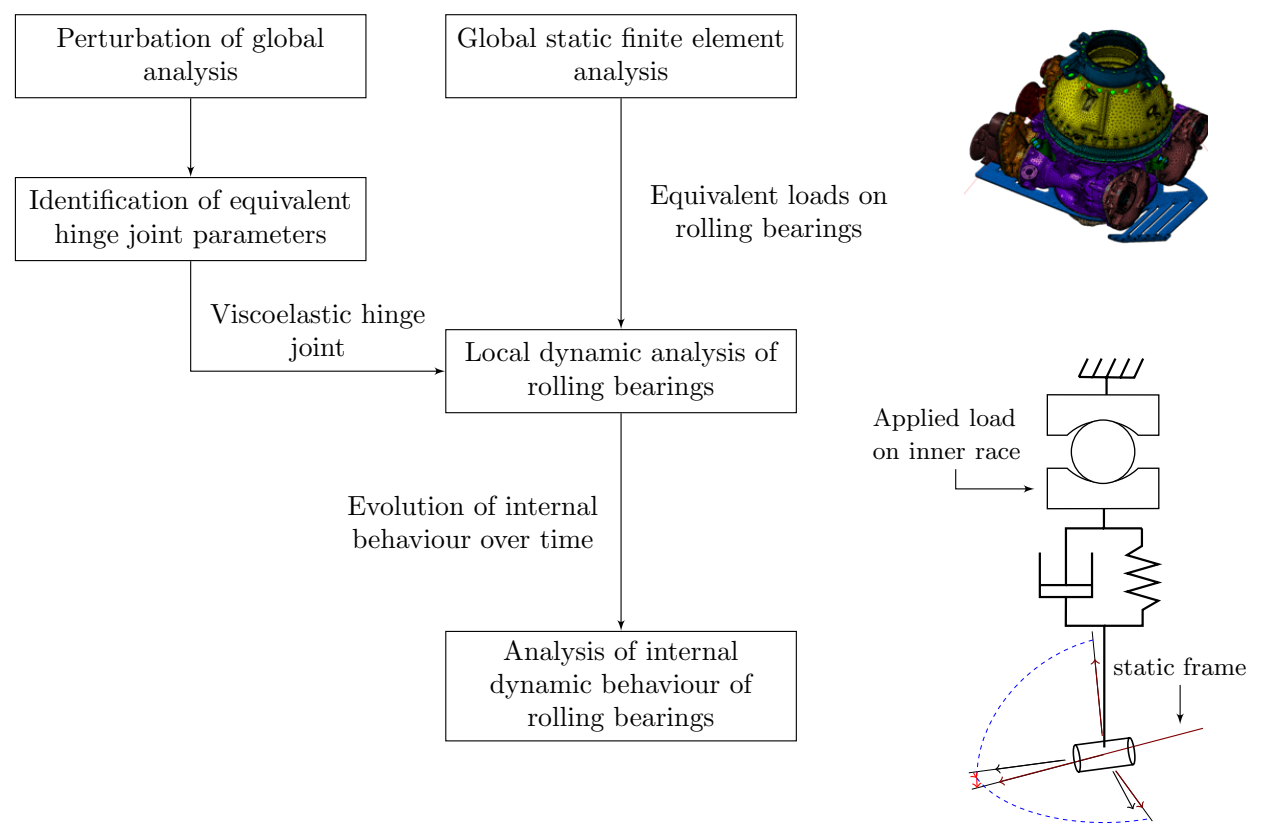

Figure 2: Modelling approach

In the proposed approach, a better estimate of the equivalent load is obtained via a global finite element analysis. Thus, a static analysis incorporating the complete system with gears and rolling bearings is carried out. Several options are available to model rolling bearings, their stiffness and geometrical defects of rolling bearings. Some interesting data on rolling bearings merge from the static analysis such as the load or pressure distribution on rolling elements, and race deformation. In this study, races are assumed to be rigid for the dynamic simulation. These data are used to reconstruct the equivalent load as seen by the rolling bearing.

Although the global finite element analysis undeniably provides a better assessment of the equivalent load, it appears from the author's experiments that, 
in its current form, the model is unable to reproduce the dynamic behaviour of a ball bearing subject only to axial and moment loads. In particular, the 150 highly perturbed behaviour of the inner race seems to be linked to the high value of the moment. This unusual behaviour is accentuated by the absence of any radial load. Also, it must be noted that allowing the inner race to move freely in the dynamic analysis is equivalent to neglecting the link between the inner race and the shaft. Indeed, if the equivalent load provided by the global 155 static analysis takes into account the rigidity of the complete system, this load is only exact at the point of static equilibrium. During the dynamic analysis, the position and the orientation of the inner race are slightly shifted from the static configuration. The value of the load imposed by the shaft on the inner race is then naturally modified. Thus the equivalent load prescribed for the inner race must be dependent on the configuration of the inner race during the dynamic analysis. This dependence must represent inertia, damping and rigidity of the rest of the system.

In this work, an additional viscoelastic hinge joint is proposed to model this dependence on the inner race configuration. The hinge joint constrains the 165 actual configuration of the inner race within a frame that corresponds to the static one. Thus, an additional force that corresponds to the rigidity of the rest of the system is considered when the position of the inner race shifts from the static solution. In the vicinity of static equilibrium, a linear viscoelastic hinge joint seems sufficient to model this additional force. The multibody dynamic 170 formulation of the hinge joint is described in more detail in Section 3.2 . Virtual work takes the form (2), where $\boldsymbol{u}$ and $\boldsymbol{\psi}$ represent the shift in position and orientation respectively.

$$
\delta \mathcal{W}_{\text {hinge }}=-\delta \boldsymbol{u}^{T} \boldsymbol{H}_{\boldsymbol{u}} \boldsymbol{u}-\delta \boldsymbol{\psi}^{T} \boldsymbol{H}_{\boldsymbol{\psi}} \boldsymbol{\psi}-\delta \boldsymbol{u}^{T} \boldsymbol{G}_{\boldsymbol{u}} \dot{\boldsymbol{u}}-\delta \boldsymbol{\psi}^{T} \boldsymbol{G}_{\boldsymbol{\psi}} \dot{\boldsymbol{\psi}}
$$

Rigidity parameters $\boldsymbol{H}_{\boldsymbol{u}}$ and $\boldsymbol{H}_{\boldsymbol{\psi}}$ are identified using a minimization process. 175 In the global finite element static analysis, an average mean displacement element is added between the inner race and its centre. Relative displacements due to various perturbation forces and moments applied to this centre of the inner ring are then computed. Minimization of the difference in behaviour between the finite element model and the equivalent hinge joint furnishes the rigidity parameters $\boldsymbol{H}_{\boldsymbol{u}}$ and $\boldsymbol{H}_{\boldsymbol{\psi}}(2)$. Unfortunately, damping parameters $\boldsymbol{G}_{\boldsymbol{u}}$ and $\boldsymbol{G}_{\boldsymbol{\psi}}$ cannot be evaluated by such a process. In this work, damping parameters are assumed to be proportional to their corresponding rigidity parameter. A coefficient of $10^{-6}$ seems to provide good results.

$$
\min _{\boldsymbol{H}_{\boldsymbol{u}}, \boldsymbol{H}_{\psi}} \sum_{i \leq n_{\text {pert }}}\left\|\boldsymbol{u}_{F E}^{i}-\boldsymbol{u}_{e q}^{i}\left(\boldsymbol{H}_{\boldsymbol{u}}, \boldsymbol{H}_{\boldsymbol{\psi}}\right)\right\|^{2}+\left\|\boldsymbol{\psi}_{F E}^{i}-\boldsymbol{\psi}_{e q}^{i}\left(\boldsymbol{H}_{\boldsymbol{u}}, \boldsymbol{H}_{\boldsymbol{\psi}}\right)\right\|^{2}
$$




\section{Description of multibody dynamic formalism}

\subsection{General description}

Various theories are available in multibody dynamic analysis such as the formalisms of the floating frame of reference, absolute nodal coordinates [18] or the finite element approach [19]. This work uses the finite element approach.

190 The configuration of a rigid body is uniquely defined by the translation of an arbitrary point and the rotation operator mapping the initial to the current configuration. Thus, it can be expressed as a time-dependent application:

$$
\mathscr{C}=\left\{(\boldsymbol{x}, \boldsymbol{R}): \mathbb{R}^{+} \mapsto \mathbb{R}^{3} \times S O(3)\right\}
$$

For rigid bodies, it is easier to use a rotation parameterization rather than working directly with the special orthogonal group $S O(3)$. Such a choice of

parameters is a crucial point since they must be able to describe rotations of large amplitude [36. Most approaches consist of expressing an arbitrary rotation in terms of elementary rotations about well defined axes such as Euler or Bryant angles. Such a geometric description provides an intuitive description of rotational motion but has the drawback that it exhibit singularity. Euler 200 parameters are also commonly used. This method is free of any singularity but it needs one redundant variable. Their use requires an additional non-linear normality constraint to be imposed. The present work employs the Conformal Rotation Vector. This parameterization seems a good choice for representing large rotations computationally. Only three independent parameters are needed.

205 Also, it has no singularity over one full rotation and has a very linear behaviour in terms of rotation angles 37. A correction procedure for the Conformal Rotation Vector is needed to allow rotation of any magnitude. Thus, each body requires the use of six parameters. The generalized coordinates of all bodies are collected in a column vector $\boldsymbol{q}$.

Joints interconnect the bodies in a multibody system. In the ideal case, these connections are expressed as algebraic equations. Kinematic constraints are distinguished into holonomic and non-holonomic constraints. Holonomic constraints $\boldsymbol{\Phi}$ are formulated as implicit functions of the generalized coordinates and time (4). Non-holonomic constraints $\boldsymbol{\Phi}_{n h}$ encountered in this work are always linear in velocities and can be expressed in the form (5) where $\dot{\boldsymbol{q}}$ notation denotes the time derivative of $\boldsymbol{q}$.

$$
\begin{gathered}
\boldsymbol{\Phi}(\boldsymbol{q}, t)=\mathbf{0} \\
\boldsymbol{\Phi}_{n h}(\boldsymbol{q}, \dot{\boldsymbol{q}}, t)=\boldsymbol{B}_{n h}(\boldsymbol{q}) \dot{\boldsymbol{q}}+\boldsymbol{g}_{n h}(t)
\end{gathered}
$$

Non-holonomic constraints are considered using a dissipation function $\mathcal{D}$, with $p$ a penalty coefficient and $k$ is scaling factor. $\boldsymbol{\lambda}$ is a set of Lagrange multipliers. The superscript ${ }^{T}$ is the transpose operator.

$$
\mathcal{D}=\frac{p}{2} \boldsymbol{\Phi}_{n h}^{T} \boldsymbol{\Phi}_{n h}+k \dot{\boldsymbol{\lambda}}^{T} \boldsymbol{\Phi}_{n h}
$$


Thus, the constrained dynamic problem is treated by augmenting the functional in Hamilton's principle. In (7), $\mathcal{L}$ is the Lagrangian of the system and $\delta \mathcal{W}$ is the virtual work of external forces that takes into account non-conservative loads.

$$
\int_{t_{1}}^{t_{2}}\left[\delta\left(\mathcal{L}-\frac{p}{2} \boldsymbol{\Phi}^{T} \boldsymbol{\Phi}-k \boldsymbol{\lambda}^{T} \boldsymbol{\Phi}-\mathcal{D}\right)+\delta \mathcal{W}\right] d t
$$

By performing the variational calculation and integrating by parts over time, the equations of motions of a constrained system are then obtained. $\boldsymbol{M}, \boldsymbol{B}$ are respectively the mass matrix and the matrix of gradients of holonomic constraints. The force vector $\boldsymbol{g}$ is the sum of the external, internal and complementary inertia forces. $\boldsymbol{r}$ is the dynamic residual vector.

$$
\left\{\begin{aligned}
\boldsymbol{r}(\boldsymbol{q}, \dot{\boldsymbol{q}}, \ddot{\boldsymbol{q}}, \boldsymbol{\lambda}, \dot{\boldsymbol{\lambda}})=\boldsymbol{M} \ddot{\boldsymbol{q}}+\boldsymbol{B}^{T}(p \boldsymbol{\Phi}+k \boldsymbol{\lambda})+\boldsymbol{B}_{n h}^{T}\left(p \boldsymbol{\Phi}_{n h}+k \dot{\boldsymbol{\lambda}}\right)-\boldsymbol{g}(\boldsymbol{q}, \dot{\boldsymbol{q}}, t) & =\mathbf{0} \\
\boldsymbol{\Phi}_{n h}(\boldsymbol{q}, \dot{\boldsymbol{q}}, t) & =\mathbf{0} \\
\mathbf{\Phi}(\boldsymbol{q}, t) & =\mathbf{0}
\end{aligned}\right.
$$

This non-linear second order differential-algebraic system (8) is solved using the HHT scheme 38]. Derived from the Newmark method, this allows a direct solution of a second order differential system. This scheme is unconditionally stable and second order accurate even in the presence of numerical dissipation. It also generates less numerical dissipation than the damped Newmark algorithm.

215 The use of these unconditionally stable schemes is of particular importance in contact problems involving friction and rolling where the dynamic response is very complex due to the large, rapidly varying contact and frictional forces applied to the system 39 .

To estimate the solution increment, the scheme requires the linearized form of the dynamic equilibrium. Knowing an approximate solution $\left(\boldsymbol{q}^{\star}, \dot{\boldsymbol{q}}^{\star}, \ddot{\boldsymbol{q}}^{\star}, \boldsymbol{\lambda}^{\star}, \dot{\boldsymbol{\lambda}}^{\star}\right)$ at time $t$ and considering a correction of the solution $(\Delta \boldsymbol{q}, \Delta \dot{\boldsymbol{q}}, \Delta \ddot{\boldsymbol{q}}, \Delta \boldsymbol{\lambda}, \Delta \dot{\boldsymbol{\lambda}})$, differentiation of (8) provides :

$$
\left[\begin{array}{cc}
\boldsymbol{M} & \mathbf{0} \\
\mathbf{0} & \mathbf{0}
\end{array}\right]\left[\begin{array}{c}
\Delta \ddot{\boldsymbol{q}} \\
\Delta \ddot{\boldsymbol{\lambda}}
\end{array}\right]+\left[\begin{array}{cc}
\boldsymbol{C}_{t} & k \boldsymbol{B}_{n h}^{T} \\
k \boldsymbol{B}_{n h} & \mathbf{0}
\end{array}\right]\left[\begin{array}{c}
\Delta \dot{\boldsymbol{q}} \\
\Delta \dot{\boldsymbol{\lambda}}
\end{array}\right]+\left[\begin{array}{cc}
\boldsymbol{K}_{t} & k \boldsymbol{B}^{T} \\
k \boldsymbol{B} & \mathbf{0}
\end{array}\right]\left[\begin{array}{c}
\Delta \boldsymbol{q} \\
\Delta \boldsymbol{\lambda}
\end{array}\right]=\left[\begin{array}{l}
-\boldsymbol{r}^{\star} \\
-\boldsymbol{\Phi}^{\star}
\end{array}\right]+O\left(\Delta^{2}\right)
$$

where $\boldsymbol{K}_{t}$ and $\boldsymbol{C}_{t}$ are the tangent stiffness and the tangent damping matrices.

$$
\boldsymbol{K}_{t}=\frac{\partial \boldsymbol{r}}{\partial \boldsymbol{q}} \quad \text { and } \quad \boldsymbol{C}_{t}=\frac{\partial \boldsymbol{r}}{\partial \dot{\boldsymbol{q}}}
$$

Finally, the non-linear equation of motion is solved iteratively by Newton's method. The exact form of the tangent operator is necessary to obtain quadratic convergence of Newton's method. However, such exactness is often too costly to obtain simply for the purposes of iteration convergence. From the author's experience, an approximate form of the tangent operators often leads to less CPU time. The computational procedure is summed up in FIG.3

\subsection{Description of a viscoelastic hinge joint}

In the rolling bearing model which has been developed, the kinematics of both races are constrained. The outer race is constrained via an ideal hinge joint 


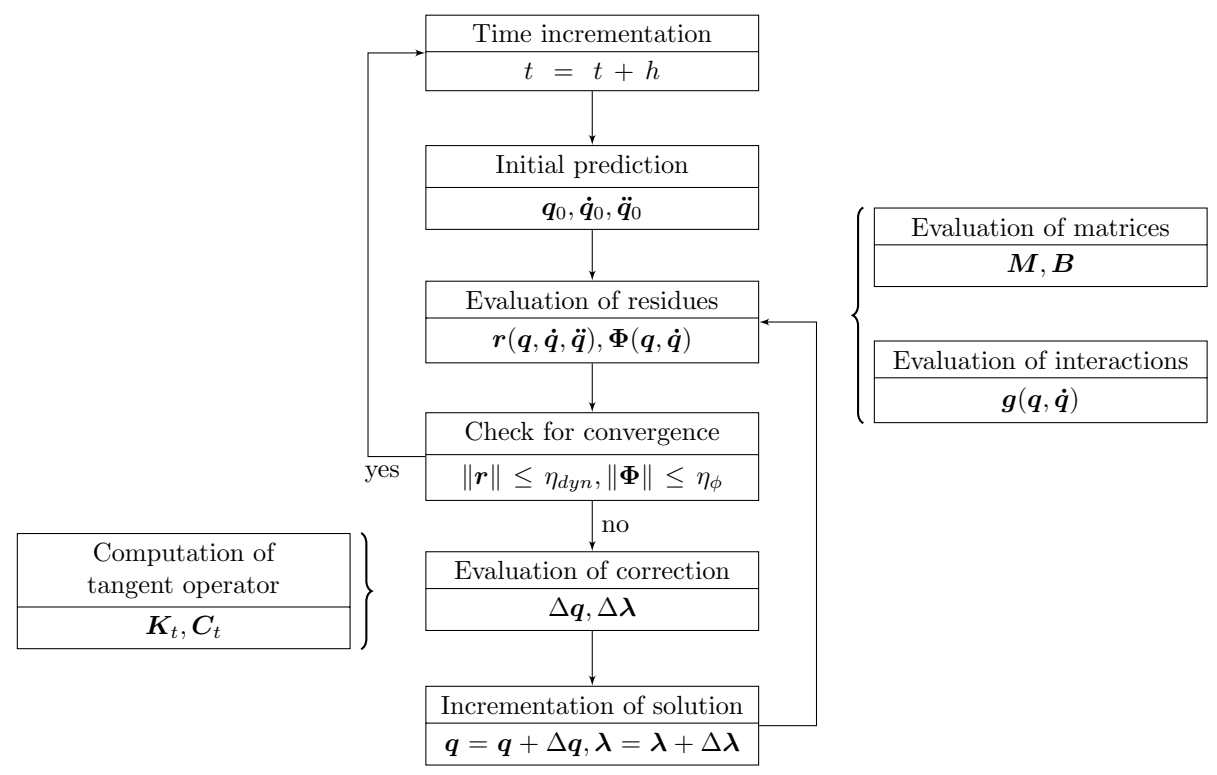

Figure 3: Iteration procedure of the implicit time integration algorithm

within an reference frame. To account for the overall stiffness of the helicopter main gearbox, the inner race is constrained via a viscoelastic hinge joint. In this section, the formulation of the viscoelastic hinge joint is derived from the ideal one.

Let $\left(\boldsymbol{\xi}_{\mathbf{1}}, \boldsymbol{\xi}_{\mathbf{2}}, \boldsymbol{\xi}_{\mathbf{3}}\right)$ and $\left(\boldsymbol{\mu}_{\mathbf{1}}, \boldsymbol{\mu}_{\mathbf{2}}, \boldsymbol{\mu}_{\mathbf{3}}\right)$ be two orthonormal triads attached to nodes $A$ and $B$, respectively, at the reference configuration (see Fig.4(a)). Let $\left(\overline{\boldsymbol{\xi}}_{1}, \overline{\boldsymbol{\xi}}_{\mathbf{2}}, \overline{\boldsymbol{\xi}}_{3}\right)$ and $\left(\overline{\boldsymbol{\mu}}_{1}, \overline{\boldsymbol{\mu}}_{2}, \overline{\boldsymbol{\mu}}_{\mathbf{3}}\right)$ be the triads obtained by mapping the reference one into the actual configuration. The corresponding rotation operators are $\boldsymbol{R}_{A}$ and $\boldsymbol{R}_{B}$ (11). Since both races are constrained within a fixed frame, the configuration of the frame $\left(A, \overline{\boldsymbol{\xi}}_{\mathbf{1}}, \overline{\boldsymbol{\xi}}_{\mathbf{2}}, \overline{\boldsymbol{\xi}}_{\mathbf{3}}\right)$ is assumed constant. Thus, variations and time variations of $\boldsymbol{x}_{A}$ and $\boldsymbol{R}_{A}$ are nil.

$$
\overline{\boldsymbol{\mu}}_{i}=\boldsymbol{R}_{A} \boldsymbol{\mu}_{i} \quad ; \quad \bar{\xi}_{i}=\boldsymbol{R}_{B} \boldsymbol{\xi}_{i}
$$

The ideal hinge joint is modelled by introducing five constraints [19]; three imposing the equality of node position

$$
\boldsymbol{x}_{A}=\boldsymbol{x}_{B}
$$

and two enforcing coaxiality of $\overline{\boldsymbol{\mu}}_{3}$ and $\overline{\boldsymbol{\xi}}_{\mathbf{3}}$.

$$
\bar{\mu}_{1}^{T} \bar{\xi}_{3}=0 \quad ; \quad \bar{\mu}_{2}^{T} \bar{\xi}_{3}=0
$$

These five constraints are usually collected in a constraint vector $\boldsymbol{\Phi}$. The constraint gradient $\boldsymbol{B}$ is then obtained by differentiating the constraint vector such 


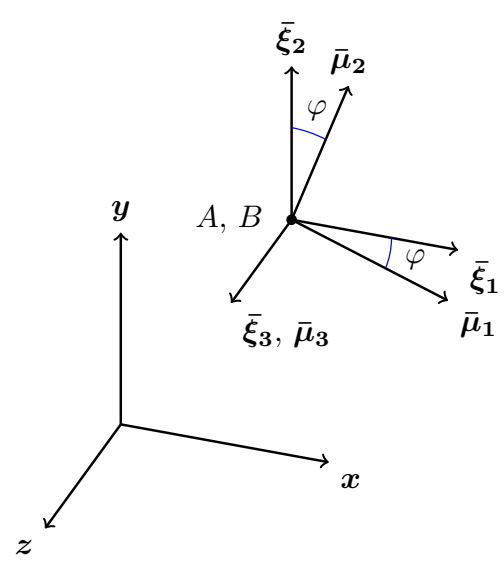

(a) Local frames to the hinge joint

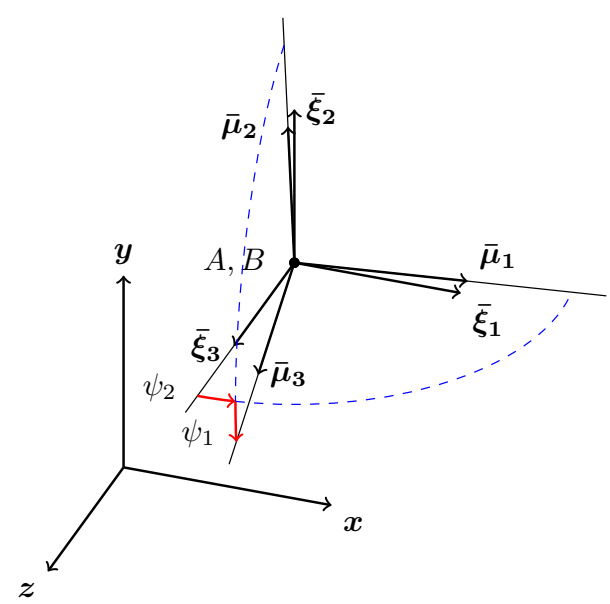

(b) Deformation kinematics of flexible hinge $\left(\boldsymbol{x}_{A} \equiv \boldsymbol{x}_{B}\right)$

Figure 4: Geometrical description of the hinge joint

that (see [19] for detailed calculations):

$$
\delta \Phi=\boldsymbol{B} \delta \boldsymbol{q}
$$

Flexible joints are not dealt with Lagrange multipliers; the elastic behaviour of the joint is associated with a strain energy $\mathcal{V}_{c}$ and the viscous behaviour is associated with non-conservative virtual work $\delta \mathcal{W}_{n c}$. Both are functions of extensional and shear strain $\boldsymbol{u}$ and bending strain $\boldsymbol{\psi}(15)$; small strains are assumed (see FIG 4(b)]. The rotation operator in $\boldsymbol{u}$ replaces strains in the natural frame of the hinge. The superscript in $\tilde{\boldsymbol{u}}$ denotes for a skew symmetric linear transformation of $\boldsymbol{u}$ equivalent to the cross product.

$$
\begin{gathered}
\boldsymbol{u}=\boldsymbol{R}_{A}^{T}\left(\boldsymbol{x}_{B}-\boldsymbol{x}_{A}\right) \quad \text { and } \quad \boldsymbol{\psi}=\left\{\begin{array}{c}
\psi_{1} \\
\psi_{2}
\end{array}\right\}=\left\{\begin{array}{r}
-\overline{\boldsymbol{\mu}}_{2}^{T} \overline{\boldsymbol{\xi}}_{3} \\
\overline{\boldsymbol{\mu}}_{1}^{T} \overline{\boldsymbol{\xi}}_{3}
\end{array}\right\} \\
\dot{\boldsymbol{u}}=\boldsymbol{R}_{A}^{T} \dot{\boldsymbol{x}}_{B} \quad \text { and } \quad \dot{\boldsymbol{\psi}}=\left\{\begin{array}{c}
\left(\tilde{\overline{\boldsymbol{\mu}}}_{2} \overline{\boldsymbol{\xi}}_{3}\right)^{T} \boldsymbol{R}_{B} \boldsymbol{\Omega}_{B} \\
-\left(\tilde{\overline{\boldsymbol{\mu}}}_{1} \overline{\boldsymbol{\xi}}_{\mathbf{3}}\right)^{T} \boldsymbol{R}_{B} \boldsymbol{\Omega}_{B}
\end{array}\right\}
\end{gathered}
$$

For the strain energy, the simplest choice may be a quadratic form, its variation being given by (17), where $\boldsymbol{H}_{\boldsymbol{u}}$ and $\boldsymbol{H}_{\boldsymbol{\psi}}$ are matrices of elastic coefficients in extension, shear and bending. In (18), $\boldsymbol{G}_{\boldsymbol{u}}$ and $\boldsymbol{G}_{\boldsymbol{\psi}}$ are matrices of viscous coefficients in extension, shear and bending. All matrices representing bending coefficients are assumed to be diagonal.

$$
\begin{gathered}
\delta \mathcal{V}_{c}=-\delta \mathcal{W}_{c}=\delta \boldsymbol{u}^{T} \boldsymbol{H}_{\boldsymbol{u}} \boldsymbol{u}+\delta \boldsymbol{\psi}^{T} \boldsymbol{H}_{\boldsymbol{\psi}} \boldsymbol{\psi} \\
\delta \mathcal{W}_{n c}=-\delta \boldsymbol{u}^{T} \boldsymbol{G}_{\boldsymbol{u}} \dot{\boldsymbol{u}}-\delta \boldsymbol{\psi}^{T} \boldsymbol{G}_{\boldsymbol{\psi}} \dot{\boldsymbol{\psi}}
\end{gathered}
$$


Finally, the contribution of the viscoelastic joint in the force vector $\boldsymbol{g}$ in $(8)$ are derived. In the expression for generalized forces, $\boldsymbol{T}_{B}$ and $\boldsymbol{\Omega}_{B}$ are respectively the tangent operator of rotation and the material angular velocity of the rotation $\boldsymbol{R}_{B}$. The iteration procedure of the implicit time integration algorithm requires the increment of generalized forces $\Delta \boldsymbol{g}_{c}$ and $\Delta \boldsymbol{g}_{n c}$. From (19|20), a rather good estimate of tangent operators can be obtained.

$$
\begin{gathered}
\boldsymbol{g}_{c}=-\left\{\begin{array}{c}
\boldsymbol{R}_{A} \boldsymbol{H}_{\boldsymbol{u}} \boldsymbol{R}_{A}^{T}\left(\boldsymbol{x}_{B}-\boldsymbol{x}_{A}\right) \\
\boldsymbol{T}_{B}^{T} \boldsymbol{R}_{B}^{T}\left(\left(\tilde{\overline{\boldsymbol{\mu}}}_{2} \overline{\boldsymbol{\xi}}_{\mathbf{3}}\right) H_{\psi, 1} \psi_{1}-\left(\tilde{\overline{\boldsymbol{\mu}}}_{1} \bar{\xi}_{\mathbf{3}}\right) H_{\psi, 2} \psi_{2}\right)
\end{array}\right\} \\
\boldsymbol{g}_{n c}=-\left\{\begin{array}{c}
\boldsymbol{R}_{A} \boldsymbol{G}_{\boldsymbol{u}} \boldsymbol{R}_{A}^{T} \dot{\boldsymbol{x}}_{B} \\
\left.\boldsymbol{T}_{B}^{T} \boldsymbol{R}_{B}^{T}\left(\left(\tilde{\overline{\boldsymbol{\mu}}}_{\mathbf{2}} \overline{\boldsymbol{\xi}}_{\mathbf{3}}\right) G_{\psi, 1}\left(\tilde{\overline{\boldsymbol{\mu}}}_{\mathbf{2}} \overline{\boldsymbol{\xi}}_{\mathbf{3}}\right)^{T}+\left(\overline{\tilde{\boldsymbol{\mu}}}_{1} \overline{\bar{\xi}}_{\mathbf{3}}\right) G_{\psi, 2}\left(\tilde{\overline{\boldsymbol{\mu}}}_{1} \overline{\boldsymbol{\xi}}_{\mathbf{3}}\right)^{T}\right) \boldsymbol{R}_{B} \boldsymbol{\Omega}_{B}\right\}
\end{array}\right.
\end{gathered}
$$

\section{Internal modelling of non-planar ball bearings}

Internal interactions are classified into two main parts, namely interaction involving rolling elements and interaction between cage and race. These interactions are treated with compliant contact models. With such models, contact forces are expressed as continuous functions of contact parameters and contact geometry. These contact parameters are computed from the positions and velocities of the contacting bodies. FIG.5 shows the internal modelling of the ball bearing. The geometric model of the ball bearing is shown in FIG.6. All geometric calculations involved are done analytically. The interaction model provides contact forces and moments.

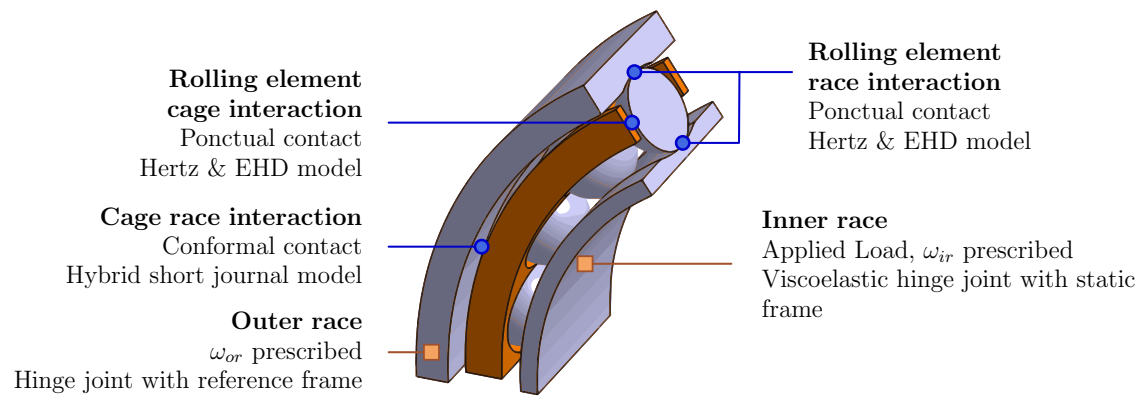

Figure 5: Ball bearing model description

\subsection{Interaction involving rolling elements}

Interaction involving rolling elements consists of interaction between balls and races and interaction between balls and cage pockets. The treatment of these rolling contacts is of primary importance since they greatly influence the dynamic behaviour of the ball bearing. 


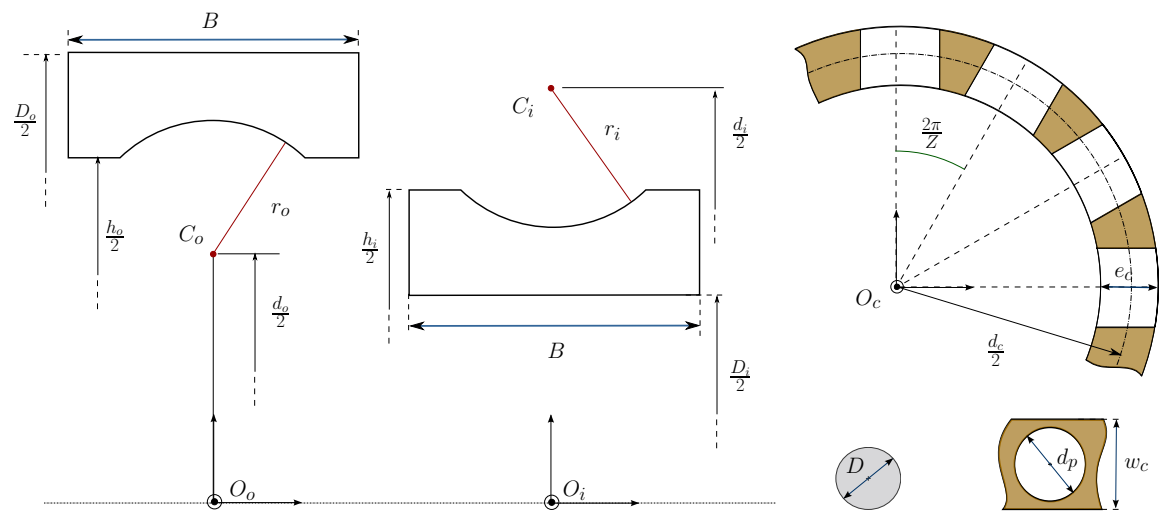

Figure 6: Geometry and dimensions of the ball bearing components

Geometric calculations. From a geometrical point of view, the former is equivalent to the geometric interaction between a torus and a sphere. The latter is characterized by the geometric interaction between a cylinder and a sphere. This simple and well known interaction is not reviewed here, and only the interaction between a torus and a sphere will be considered. FIG.7 shows the contact configuration. Let $\left(O_{i}, \boldsymbol{X}_{\boldsymbol{i}}^{\mathbf{0}}, \boldsymbol{Y}_{\boldsymbol{i}}^{\mathbf{0}}, \boldsymbol{Z}_{\boldsymbol{i}}^{\mathbf{0}}\right)$ be an orthonormal triad attached to the inner race such that $\boldsymbol{Z}_{\boldsymbol{i}}^{\mathbf{0}}$ is coincident with the inner race axis. Let the coordinates of the ball centre $O_{k}$ in this triad be $\left(\hat{X}_{k}, \hat{Y}_{k}, \hat{Z}_{k}\right)$. The potential contact between the torus and the sphere is necessarily contained in the plane that passes through $O_{k}$ and includes the line $\left(O_{i}, \boldsymbol{Z}_{\boldsymbol{i}}^{\mathbf{0}}\right)$. Let us define a new frame $\left(O_{i}, \boldsymbol{X}_{\boldsymbol{i}}, \boldsymbol{Y}_{\boldsymbol{i}}, \boldsymbol{Z}_{\boldsymbol{i}}\right)$ rotated about $\boldsymbol{Z}_{\boldsymbol{i}}^{\mathbf{0}}$ such that $\left(O_{i}, \boldsymbol{X}_{\boldsymbol{i}}, \boldsymbol{Z}_{\boldsymbol{i}}\right)$ is the plane that contains the potential contact. The magnitude of the rotation is

$$
\gamma=\arctan \left(\frac{\hat{Y}_{k}}{\hat{X}_{k}}\right)
$$

Considering the triangle drawn in FIG.7, the contact angle $\alpha$ and the contact deflection $\delta$ can be easily obtained (22) (23). A negative $\delta$ indicates no contact between the ball and the race. The contact between the outer race and the rolling elements can be expressed in a very similar form. Knowing the location of the contact point and the contact deflection, all the geometric and kinematic data required by the contact model are available.

$$
\begin{gathered}
\tan \alpha=\frac{\hat{Z}_{k}}{\frac{d_{i}}{2}-\sqrt{\hat{X}_{k}^{2}+\hat{Y}_{k}^{2}}} \\
\delta=\sqrt{\hat{Z}_{k}^{2}+\left(\frac{d_{i}}{2}-\sqrt{\hat{X}_{k}^{2}+\hat{Y}_{k}^{2}}\right)^{2}}+\frac{D}{2}-r_{i}
\end{gathered}
$$




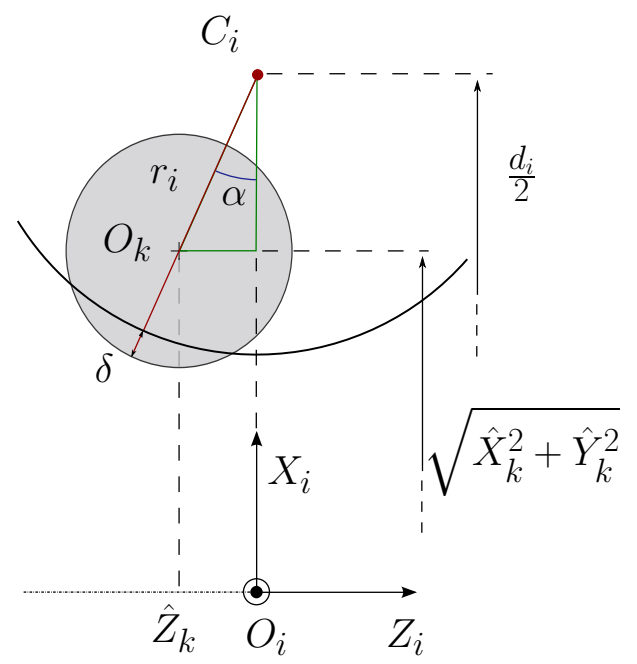

Figure 7: Geometrical interaction between the inner race and a ball

Rolling contact model. The contact force and moment calculations of rolling contacts are based on the elastohydrodynamic theory of lubrication. In this work coupling between normal and tangential contact problems is neglected. The normal load is computed first. Since an elastohydrodynamic pressure profile is similar to the hertzian profile, it is reasonable to use the classical hertzian point contact solution. Thus, the normal load $F_{n}$ is linked with the contact deflection $\delta$ by (24). $k_{h}$ is the contact rigidity which depends on the curvature of the contacting surfaces and the material properties of contacting bodies. More information about Hertz contact theory can be found in [40].

$$
F_{n}=k_{h} \delta^{3 / 2}
$$

The fluid film thickness and lubricant traction are computed. The fluid film thickness may be estimated using the explicit formula proposed by $\mathrm{Ni}$ jenbanning et al. [32, 33. Lubricant traction is computed using the work of Jacod [34. The elastohydrodynamic lubrication contacts are assumed smooth. If isothermal conditions are assumed, an explicit formula is available for the lubricant coefficient of friction. Otherwise, the estimate of lubricant traction requires the solution of a non-linear equation to compute the temperature increase of the fluid film. A WLF model [41] describes the lubricant rheology. The non-Newtonian behaviour of the lubricant is modelled using the Eyring model. The fluid used in this study corresponds to the NATO O-156 specification. Fig 8 
compares the friction coefficient of several models. Under isothermal conditions, the friction coefficient is slightly overestimated. In the legend of FIG.8, the adjective approximate refers to the way which the pressure-viscosity coefficient is calculated 41. Considering the huge number of simulated contacts and the relatively small discrepancies between the different models, isothermal conditions and approximate value of the pressure-viscosity coefficient are used.

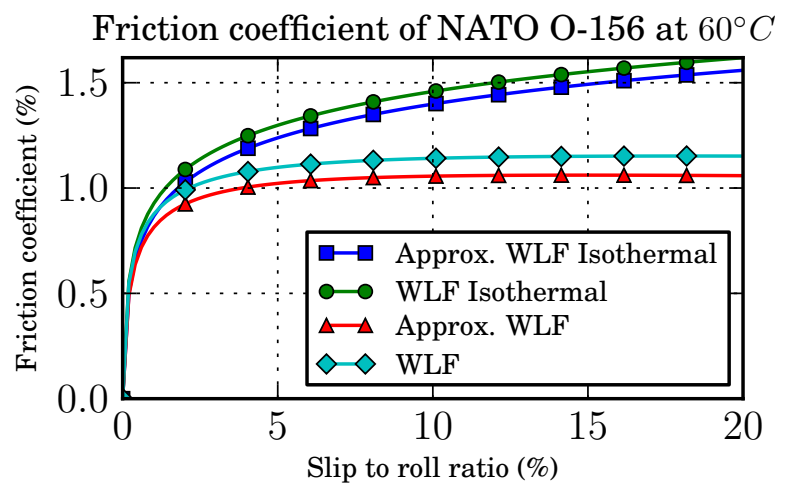

Figure 8: Typical friction coefficient, comparison between various models.

\subsection{Interaction between race and cage}

This paper is concerned with the modelling of ball bearings of helicopter main gearboxes. In such bearings, the cage is centred on the outer race. If the interaction between the cage and the race is mainly hydrodynamic, then due to the high moment prescribed, metal-to-metal contact may occur. Thus, 270 a transition model has been developed to ensure continuity of contact forces between the different regimes. This model is based on the work of Flores et al. 20, 22].

Hydrodynamic model. A commonly accepted approach is to employ the theory of lubrication for journal-bearings Excellent reviews of the theoretical background and basic principles are available in specialized books such as Pinkus and Sterlicht 42] or Frêne et al. [43]. The pressure distribution of thin viscous fluid films is governed by the Reynolds Equation. Analytical solutions of this partial differential equation can be obtained via several assumptions on fluid flow and boundary conditions. The hydrodynamics of the race cage contact is simulated by the well-known short journal bearing solution 42. The short journal bearing solution is derived by neglecting circumferential variations in the fluid pressure and assuming Gümbel boundary conditions for the pressure. The Gümbel conditions, which account for the film breakdown, take into account the existence of a zero pressure zone. Even though it leads to a violation of the continuity of the fluid flow in the film, the Gümbel conditions furnish better predictions of the load capacity of the journal bearing. The short journal model 


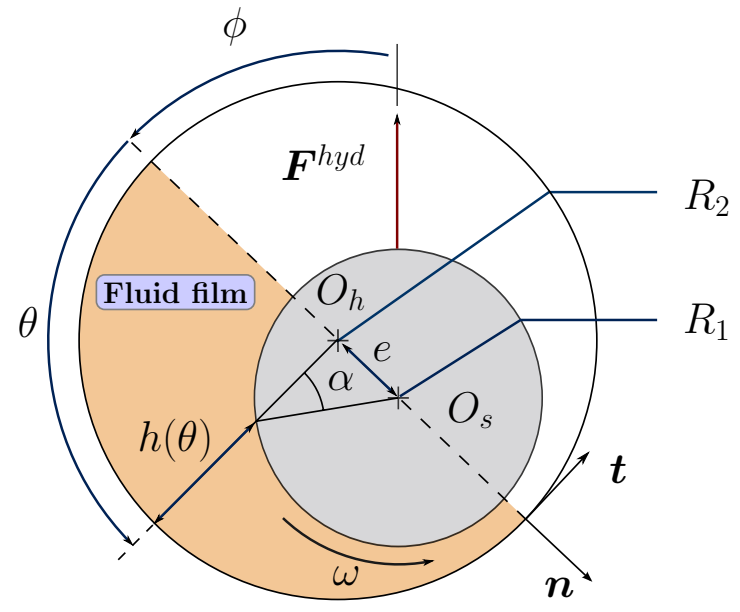

Figure 9: Cross section of a journal bearing

is justified for a length-to-diameter ratio $L / D \leq 1 / 8$. In practice, this solution is used until $L / D \leq 1 / 2$. The different notations are presented in FIG 9. Considering the small radial clearance between the cage and the guiding race, the influence of any misalignment is not taken into account in the journal bearing model. Also any dynamic effect due to variations in eccentricity is neglected. The force components of the fluid film for short journal bearing are written as:

$$
\left\{\begin{array}{l}
F_{n}^{h y d}=\frac{\mu \omega R L^{3}}{C^{2}} \frac{\varepsilon^{2}}{\left(1-\varepsilon^{2}\right)^{2}} \\
F_{t}^{h y d}=\frac{\pi}{4} \frac{\mu \omega R L^{3}}{C^{2}} \frac{\varepsilon \sqrt{1-\varepsilon^{2}}}{\left(1-\varepsilon^{2}\right)^{2}}
\end{array}\right.
$$

In the above equation, $\varepsilon=e / C$ is the relative eccentricity, $C=R_{2}-R_{1}$ is the radial clearance, $L$ is the journal length and $R$ is the mean journal radius. 275 The magnitude of the hydrodynamic force is linearly dependent on the dynamic viscosity $\mu$ and the sum of the rotational speeds $\omega$. The friction torque is given by integrating the fluid shear stress on the shaft or bearing surface (26). In the case of a ball bearing with outer race guidance, the cage plays the role of the shaft whereas the outer race is the equates to the bearing.

$$
C^{h y d}=\frac{\pi \mu \omega R^{3} L}{C} \frac{2+\varepsilon}{(1+\varepsilon) \sqrt{1-\varepsilon^{2}}}
$$

Dry contact model. When the relative eccentricity is greater than unity, metalto-metal contact occurs. Unlike point contact, Hertz theory does not provide a uniquely defined load-deflection relationship for cage-race interaction. The treatment of the metallic interaction follows that proposed in [7, and employs a 
Lundberg empirical load-deflection relationship to determine normal load. From

a prescribed contact deflection, the method proposed by Lundberg requires the solution of a non-linear equation to compute the contact load $F_{n}^{d r y}$. A good initial guess was put forward by Palmgren (see [7 p.38-39). The tangential force $F_{t}^{d r y}$ is then obtained assuming complete slip of the contact area.

Hybrid short journal model. Both interaction models for the hydrodynamic regime and metal-to-metal contact have been presented. Examining (25) and (26), it appears that when the fluid film thickness becomes very thin, i.e. the journal is very close to the bearing surface, the force and torque due to the lubricant tend to infinity (27). As $\boldsymbol{F}^{d r y}$ is zero at the beginning of the contact, a simple transition between the hydrodynamic and the dry interaction model will inevitably create a discontinuity in the contact relationship. This discontinuity inadmissible and leads to absurd results in the dynamic analyses of ball bearings.

$$
\left\{\begin{array}{lll}
F_{n}^{\text {hyd }} & \underset{\varepsilon \rightarrow 1}{\sim} \frac{\mu \omega R L^{3}}{C^{2}} \frac{1}{\left(1-\varepsilon^{2}\right)^{2}} \\
F_{t}^{\text {hyd }} & \underset{\varepsilon \rightarrow 1}{\sim} \frac{\pi}{4} \frac{\mu \omega R L^{3}}{C^{2}} \frac{1}{\left(1-\varepsilon^{2}\right)^{3 / 2}}
\end{array}\right.
$$

To avoid numerical instability and to ensure continuity of the contact relationship, Flores et al. 20, 22] proposed the definition of a boundary layer. In this layer, the contact force is a weighted average of hydrodynamic and metallic forces. A physical interpretation of the boundary layer may be that layer in which the lubrication regime becomes mixed due to surface asperities. The layer is defined by a size $e_{0}$, the corresponding relative size being defined by $\varepsilon_{0}=e_{0} / C$. The choice of $e_{0}$ must made carefully. Too high a value may greatly affect the behaviour of the model, while discontinuities may reappear with too small a one. A typical value for $\varepsilon_{0}$ is $5 \%$. Examining (27), a linear weighting function seems insufficient to remove the discontinuity. The present model makes use of a different function weighting function $f_{F}$.

$$
f_{F}(\varepsilon)=\exp \left(\frac{2 \chi(\varepsilon)}{\chi(\varepsilon)-1}\right) \quad ; \quad \chi(\varepsilon)=\frac{\varepsilon-1}{\varepsilon_{0}}
$$

For the torque, the linear function $f_{C}$. propose by Flores et al. is used.

$$
f_{C}(\varepsilon)=\frac{1+\varepsilon_{0}-\varepsilon}{\varepsilon_{0}}
$$

Finally, the approach is summarized in 30,31 . It should be noted that the clearance $C$ used for the hydrodynamic force model is replaced by $C+e_{0}$ in the hybrid model. Fig 10 shows a typical form of the contact force of the hybrid 
model.

$$
\begin{aligned}
& \boldsymbol{F}^{\text {total }}= \begin{cases}\boldsymbol{F}^{\text {hyd }} & \text { if } \quad \varepsilon \leq 1 \\
f_{F}(\varepsilon) \boldsymbol{F}^{h y d}+\left(1-f_{F}(\varepsilon)\right) \boldsymbol{F}^{d r y} & \text { if } \quad 1<\varepsilon \leq 1+\varepsilon_{0} \\
\boldsymbol{F}^{\text {dry }} & \text { if } \quad \varepsilon>1+\varepsilon_{0}\end{cases} \\
& \boldsymbol{C}^{\text {total }}= \begin{cases}\boldsymbol{C}^{\text {hyd }} & \text { if } \quad \varepsilon \leq 1 \\
f_{C}(\varepsilon) \boldsymbol{C}^{\text {hyd }}+\left(1-f_{C}(\varepsilon)\right) \boldsymbol{C}^{d r y} & \text { if } \quad 1<\varepsilon \leq 1+\varepsilon_{0} \\
\boldsymbol{C}^{\text {dry }} & \text { if } \quad \varepsilon>1+\varepsilon_{0}\end{cases}
\end{aligned}
$$

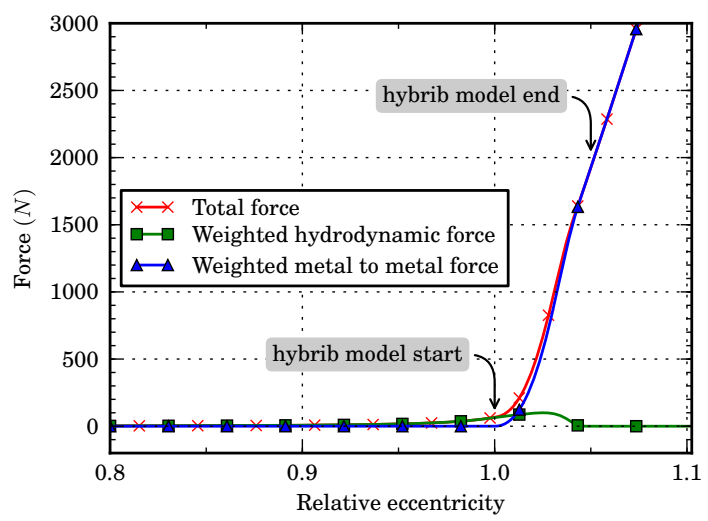

(a) Force of the hybrid contact model

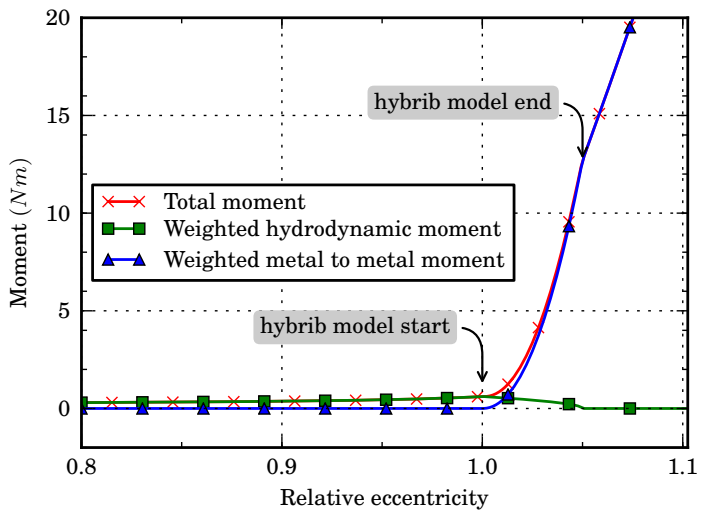

(b) Moment on ball separator for the hybrid contact model

Figure 10: Hybrid conformal contact model data 


\subsection{Internal interaction in a multibody dynamic context}

290 Models of internal interactions that occur in a ball bearing have been presented. These models have to be replaced in the context of a multibody dynamic formalism. In particular, the implicit time integration procedure, which ensures unconditional stability requires the computation of tangent operators $\boldsymbol{K}_{t}$ and $\boldsymbol{C}_{t}$ (10). Thus, an estimate of the variation of contact forces must be provided.

Rolling contact. Regarding rolling contacts, the normal force depends only on the generalized coordinates whereas $\boldsymbol{F}_{\boldsymbol{T}}$ depends also the derivatives of the generalized coordinates. The normal load $F_{n}$ is expressed in the form (32). Again, $\delta$ is the contact deflection. The contact rigidity $k_{h}$ depends on the curvature of contacting surfaces and material properties. Finally, the contact force $\boldsymbol{F}$ is expressed in the form (33) where $\boldsymbol{n}$ is the normal vector.

$$
\begin{gathered}
F_{n}(\boldsymbol{q})=k_{h}(\boldsymbol{q}) \delta(\boldsymbol{q})^{3 / 2} \\
\boldsymbol{F}(\boldsymbol{q}, \dot{\boldsymbol{q}})=F_{n}(\boldsymbol{q}) \boldsymbol{n}(\boldsymbol{q})+\boldsymbol{F}_{\boldsymbol{T}}(\boldsymbol{q}, \dot{\boldsymbol{q}})
\end{gathered}
$$

The contribution of the normal load in the tangent stiffness operator is computed from (34). In this expression, the influence variation in contact rigidity is neglected. Since geometrical interactions are computed analytically, explicit expressions of $\delta$ and $\boldsymbol{n}$ are available, and an approximate explicit form of this contribution is thus obtained.

$$
\begin{aligned}
\Delta \boldsymbol{F}_{\boldsymbol{n}}(\boldsymbol{q}) & =\Delta F_{n}(\boldsymbol{q}) \boldsymbol{n}(\boldsymbol{q})+F_{n}(\boldsymbol{q}) \Delta \boldsymbol{n}(\boldsymbol{q}) \\
& \simeq \frac{3}{2} k_{h}(\boldsymbol{q}) \delta(\boldsymbol{q})^{\frac{1}{2}} \Delta \delta(\boldsymbol{q}) \boldsymbol{n}(\boldsymbol{q})+F_{n}(\boldsymbol{q}) \Delta \boldsymbol{n}(\boldsymbol{q})
\end{aligned}
$$

In the EHD lubrication model, the contribution of the tangential component is more cumbersome to derive. The approach presented in [31 is used again.

Conformal contact. The force and torque furnished by the hybrid short journal model depends on the generalized coordinates and their derivatives.

$$
\boldsymbol{F}^{\text {total }}=\boldsymbol{F}^{\text {total }}(\boldsymbol{q}, \dot{\boldsymbol{q}}) \quad ; \quad \boldsymbol{C}^{\text {total }}=\boldsymbol{C}^{\text {total }}(\boldsymbol{q}, \dot{\boldsymbol{q}})
$$

Their contribution to the tangent stiffness and tangent damping matrices are easily achieved once the variation of the contact deflection and the relative eccentricity have been expressed. The differentiation of closed-form expression (25 26) gives the influence of the hydrodynamic force. For the dry contact, the finite difference of the Lundberg empirical formula gives a rather good estimate. It should be noted that the during the transition phase, the variation of the weight functions $\left(f_{F}, f_{C}\right)$ has to be considered. 


\begin{tabular}{ccc}
\hline Dim. $(\mathrm{mm})$ & Outer race & Inner race \\
\hline$d$ & 157.3 & 158.4 \\
$r$ & 9.906 & 9.753 \\
$D$ & 196. & 130. \\
$h$ & 166. & 150. \\
$B$ & 34. & 34. \\
\hline \hline \multicolumn{2}{c}{ Rolling elements } \\
\hline \hline & \multicolumn{2}{c}{19.05} \\
\hline \hline$d_{c}$ & \multicolumn{2}{c}{ Ball separator } \\
$d_{p}$ & 20.23 \\
$e_{c}$ & \multicolumn{2}{c}{7.42} \\
$w_{c}$ & 25.4 \\
$Z$ & 22 \\
\hline
\end{tabular}

Table 1: Dimensions of the ball bearing

\section{Results and discussion}

The approach presented in this paper has been applied to the dynamic analysis of a ball bearing whose dimensions are summarized in ТАВ 1 . Balls and races are made of steel, and the cage of bronze. As can be seen in TAB,1 the cage is guided by the outer race in this example.

Two different load cases have been considered, both having the specific features described previously. Thus, only thrust load and moment are present. The only difference between the load cases is the value of the moment. With a moderate value of moment load, load case (a) may be associated with a fairly good behaviour of the ball bearing. The high value of moment load in the second load case will lead to rapid failure of the ball bearing. The second load case has been experienced on a test rig, and experimental observations have been compared with the numerical results.

The boundary conditions used are summarized in TAB 2, The outer race is fixed rigidly within the housing. The rotation velocity of the inner race is 3060 $\mathrm{rev} / \mathrm{min}$. As previously explained, the inner race is linked to the static reference frame via a viscoelastic hinge joint. Rigidity and damping parameters of the joint have been identified using global finite element simulations (see Section 2). Perturbation forces and moments have been applied in the three directions to identify the rigidity parameters of the joint. Their magnitudes lay in the ranges $[-1000 N ; 1000 N]$ and $[-1000 N m ; 1000 N m]$ respectively. Elastic coefficients in extension and shear are equal to $10^{6} \mathrm{~N} / \mathrm{mm}$, and bending coefficients are close to $10^{5} \mathrm{Nm} / \mathrm{rad}$. The viscous coefficients matrices are assumed proportional to the elastic coefficients matrices, and the scale factor is $10^{-6}$.

The internal modelling of the ball bearing is described in Section 4 . The fluid used in this study corresponds to the NATO O-156 specification. The operating temperature is assumed to be $100^{\circ} \mathrm{C}$, this choice being based on experimental 


\begin{tabular}{ccc}
\hline \multicolumn{3}{c}{ Prescribed load on inner race } \\
\hline Load case & case (a) & case (b) \\
\hline Axial load & $34000 \mathrm{~N}$ & $34000 \mathrm{~N}$ \\
Radial load & $0 \mathrm{~N}$ & $0 \mathrm{~N}$ \\
Moment load & $100 \mathrm{Nm}$ & $430 \mathrm{Nm}$ \\
\hline \hline \multicolumn{2}{c}{ Prescribed } & rotation speed \\
\hline Inner race & $3060 \mathrm{rev} / \mathrm{min}$ \\
Outer race & $0 \mathrm{rev} / \mathrm{min}$ \\
\hline
\end{tabular}

Table 2: Boundary conditions for dynamic ball bearing simulation

observations.

In both cases, a quasi-static simulation provides initial values of the generalized coordinates. Initial velocities are obtained assuming pure rolling at the contact points. The HHT method is used to integrate the equation of motion. The damping parameter $\alpha_{f}$, which must lie within $\left(0, \frac{1}{3}\right]$ is chosen to be 0.1 . The time step is selected automatically. The automatic step size control is based on the computation of an estimate of the local truncation error (see ch.11 in [19]). For this simulation the time step was initialized at $10^{-6} s$, and lay between $10^{-8} s$ and $210^{-6} s$. The absolute unbalance allowed at each time 340 step is $10^{-6}$. For constraints, the convergence threshold is $10^{-8}$. In both load cases, the dynamic simulations were carried out over $0.8 \mathrm{~s}$ corresponding to approximately 40 revolutions of the inner ring. It was found that this period of time was sufficient to provide a fairly well-defined steady-state behavior of the bearing. The following sections present some results provided by the dynamic analysis of the ball bearing tested. Cage motion is investigated with special care since this provides a good picture of ball bearing dynamic performance. Cage whirl velocity and orbit shapes are the key parameters used to assess the overall cage motion. Also, the evolution over time of the interaction between cage and outer race is shown.

\subsection{Results for case (a)}

FIG 11 shows the load distribution on the rolling elements and their corresponding contact angle. Since in this case the thrust load is predominant, the variation of contact angle is small. For both inner and outer contacts, the variation is less than $5^{\circ}$. The magnitude of the outer race contact is slightly 355 greater than for the inner race contact because of inertial forces. It should be noted that rolling elements are heavily loaded, and the intensity of the impacts between balls and cage may therefore be significant.

The whirl orbit is plotted in a plane normal to the bearing axis in FIG 12(a) The dashed circle represent the guide clearance; hence if the cage centre lies on this circle the cage will be constantly touching the race at the guide land. There is no cage whirl at all, but rather the mass centre of the cage experiences an erratic motion. This observation seems in accordance with a previous study by Gupta who analyzed the cage dynamic behaviour of radially loaded bearings 
[44. It was observed that when the load is fixed in direction, whirl motion is absent, resulting in reduced cage interaction. In this study, the moment load is stationary, so no cage whirl was expected. The cage rotation velocity is plotted in FIG.12(b) The variation in cage rotation velocity is rather small and represents only $1.45 \%$ of its mean value.

The phase portrait of the inner ring and cage are plotted in FIG.13. In the 370 horizontal and vertical directions, the inner race motion is quasi-periodic. The perturbed oscillatory motion is excited by the prescribed rotation speed and the stationary moment load. In the axial direction however, the phase portrait shows a fixed attractor close to the point $(0.3470,0$. $)$. The inner ring quickly reaches this state. The erratic motion of the cage is again visible in FIG 13(b) 375 In all directions, the motion is chaotic.

Overall cage interactions can be discussed further in terms of the variations in contact load at the guide lands and in the cage pockets. FIG.14 shows the variation in contact load at the guide lands. Impacts on cage pockets are shown in FIG.15 The cage inevitably hits the guiding race several times, but the magnitude of contact forces is limited. The distinct collision peaks correspond to the erratic motion of the cage shown on FIG.12(a). Because of small pocket clearances, cage pocket impacts are frequent. Most impacts have a magnitude less than $150 N$. Some long duration contacts of very small amplitude can also be distinguished in FIG 15(a) In FIG 15(b) it can be observed that cage pocket 385 impacts do not occur in any particular orbital area. Finally, the ball bearing behaviour in terms of cage dynamics and cage interaction is satisfactory in this case. This was to be expected since the moment load is of moderate magnitude. 


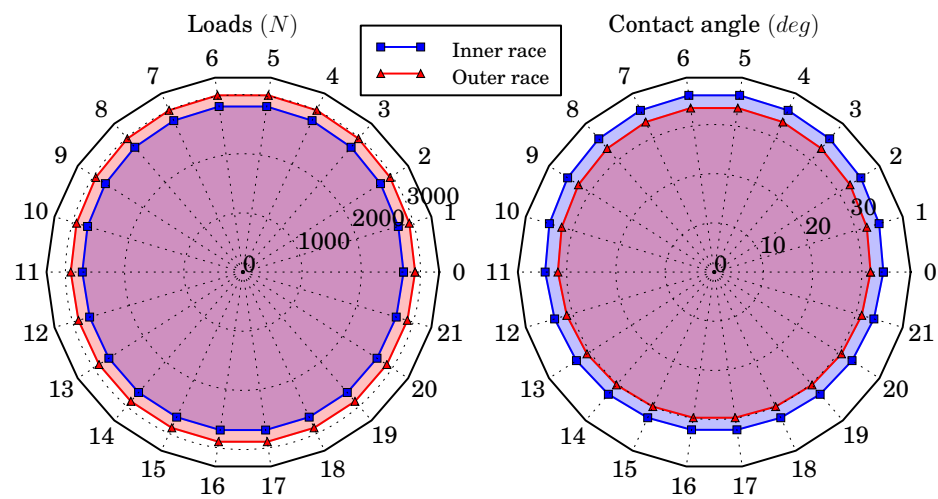

Figure 11: Rolling element load distribution and corresponding contact angle, quasi-static simulation, case (a)
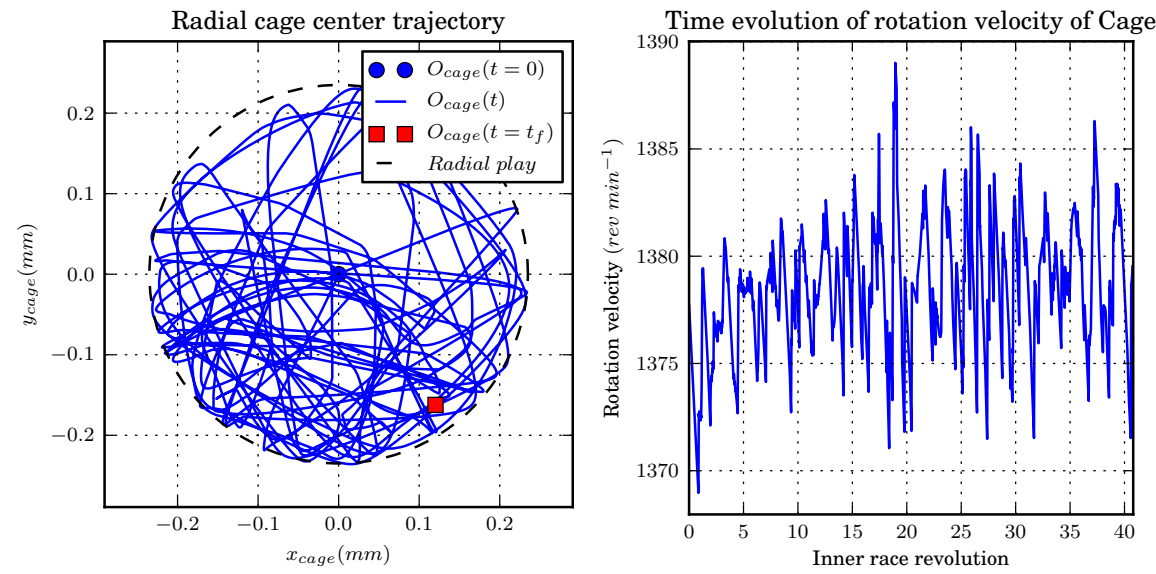

(a) Trajectory of the cage mass centre

(b) Evolution over time of the cage velocity

Figure 12: Trajectory and rotation velocity of the cage, case (a) 

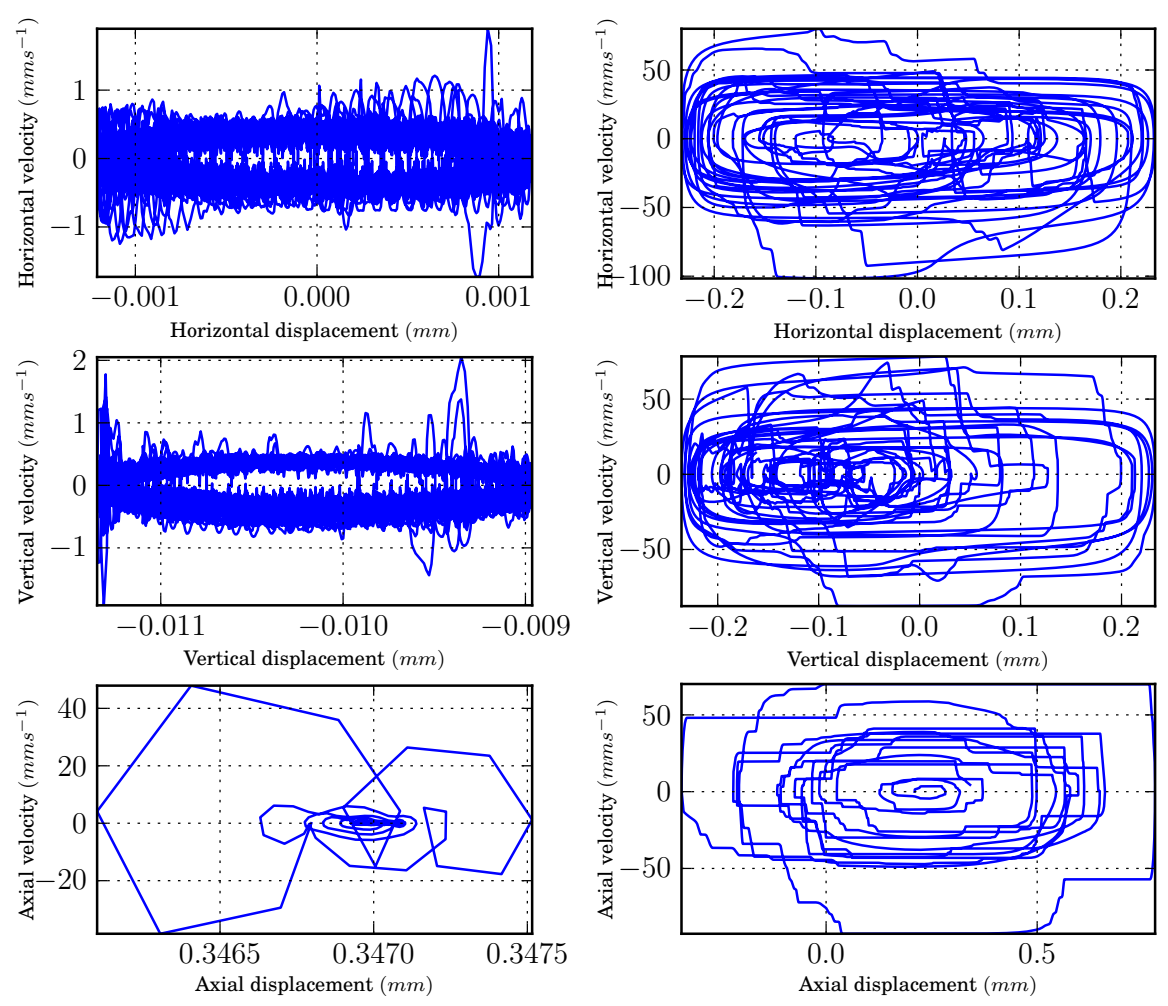

(a) Phase portrait of the inner ring

(b) Phase portrait of the cage

Figure 13: Phase portrait of inner ring and ball separator, case (a) 


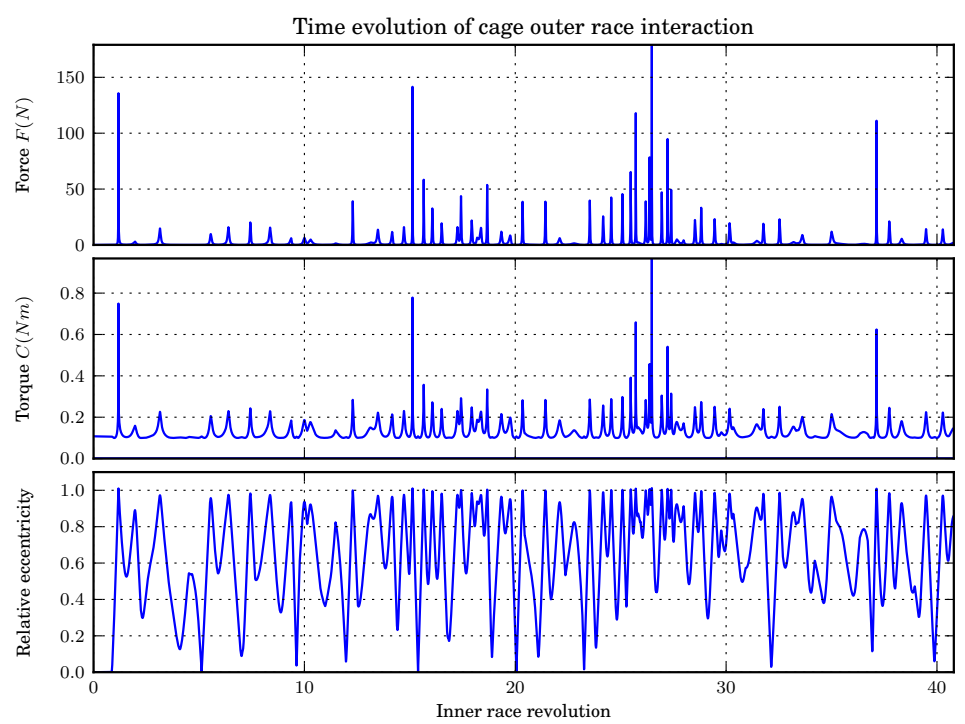

Figure 14: Evolution over time of conformal contact data between the cage and outer race, case (a)

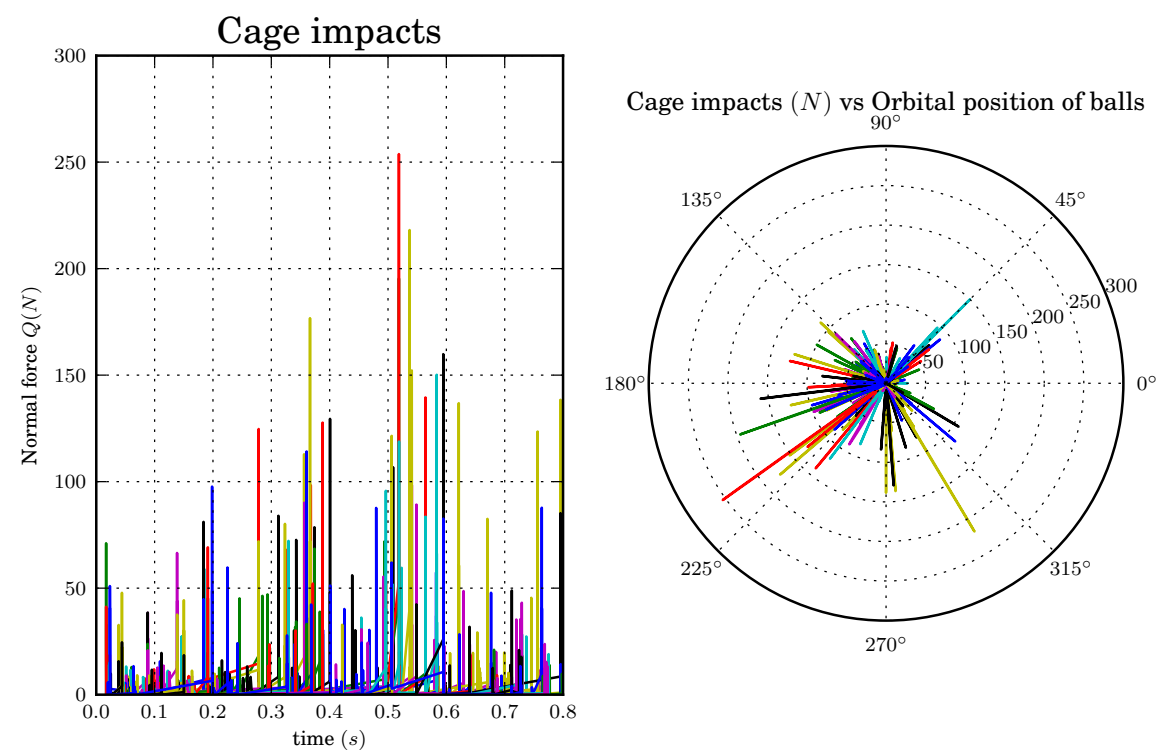

(a) Evolution over time of impacts on the (b) Impacts plotted as a function of the orcage bital position of the balls

Figure 15: Impacts on the cage, case (a) 


\subsection{Results for case (b)}

The load distribution on rolling elements and the corresponding contact angle are plotted in Fig.16. The high value of moment load leads to a large variation of contact load and contact angle. For both inner and outer contacts, variation in contact angle is about $15^{\circ}$ leading to an excessive rolling element excursion. Such a circumferential excursion results in strong interactions with the cage and cage motion is thereby affected.

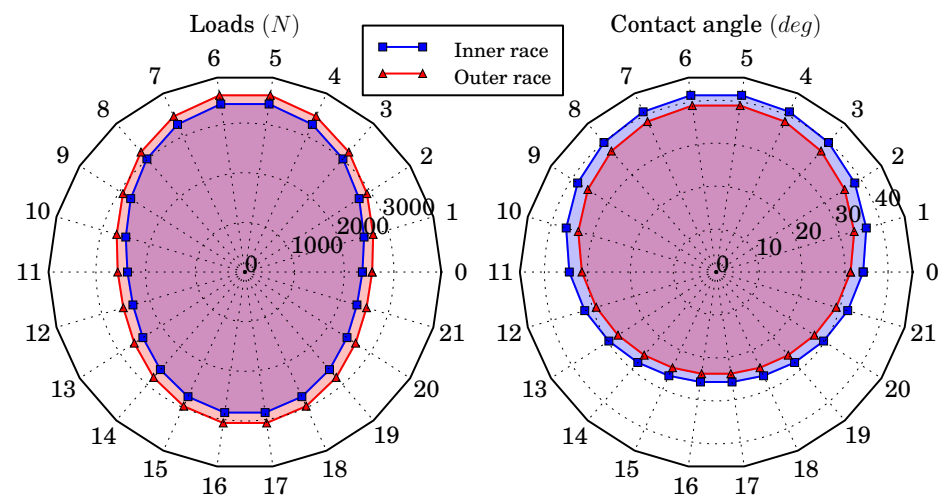

Figure 16: Rolling element load distribution and corresponding contact angle, quasi-static simulation, case (b)

395

the cage is constantly crushed against the outer race. The contact remains within a well defined area. The cage centre position slightly exceeds the radial play, so metal-to-metal contact occurs. Here, the utility of the continuous contact force model for the hybrid short journal model is highlighted. Thus, the equations of motion can be integrated even with an implicit scheme. If the cage trajectory obviously shows a malfunction of the ball bearing, with a relative variation about $1.4 \%$, the cage rotation velocity fails to provide any valuable information on the dynamic performance of the ball bearing (see FIG.17(b)].

The inner race again exhibits a quasi-periodic motion in the horizontal and vertical directions (see FIG 18(a)). The perturbation of the oscillating motion is of the same order of magnitude as in the first case. In the axial direction, the inner ring rapidly approaches to the point $(0.3425,0$.) with a gentle oscillation around it. In the phase portrait of the ball separator displayed in FIG 18(b) the almost constant radial position of the cage mass centre is also visible. In axial position $z=0.2 \mathrm{~mm}$. In this model, since the hybrid short journal model produces no axial force, the axial motion of the cage is induced only by ball impacts on pockets. Thus, in the axial direction, the absence of viscous forces explains this oscillatory behaviour. 


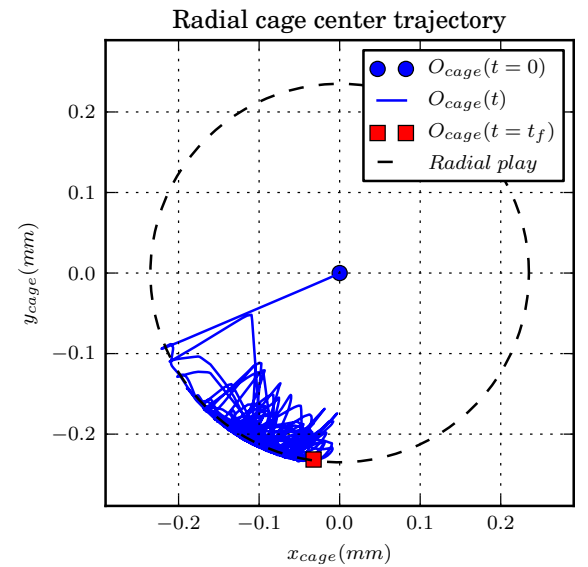

(a) Trajectory of the cage mass centre

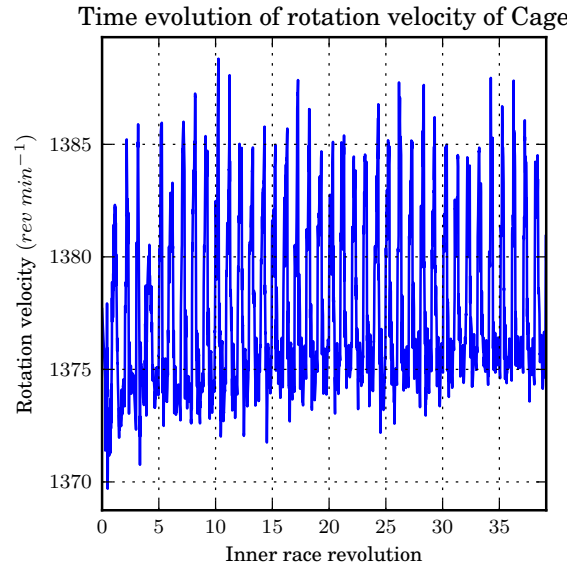

(b) Evolution over time of the cage velocity

Figure 17: Trajectory and rotation velocity of the cage, case (b)
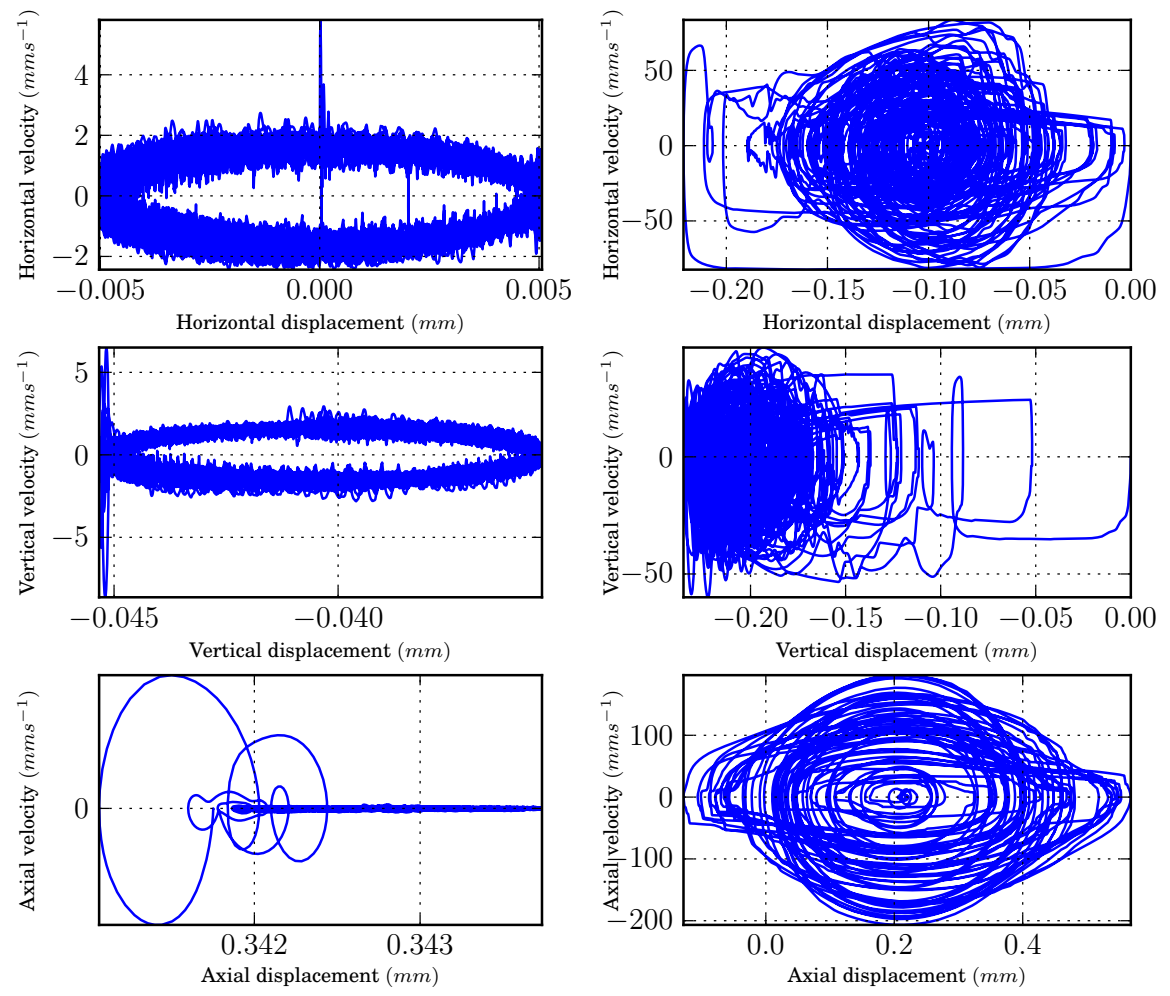

(a) Phase portrait of the inner ring

(b) Phase portrait of the cage

Figure 18: Phase portrait of inner ring and cage, case (b) 

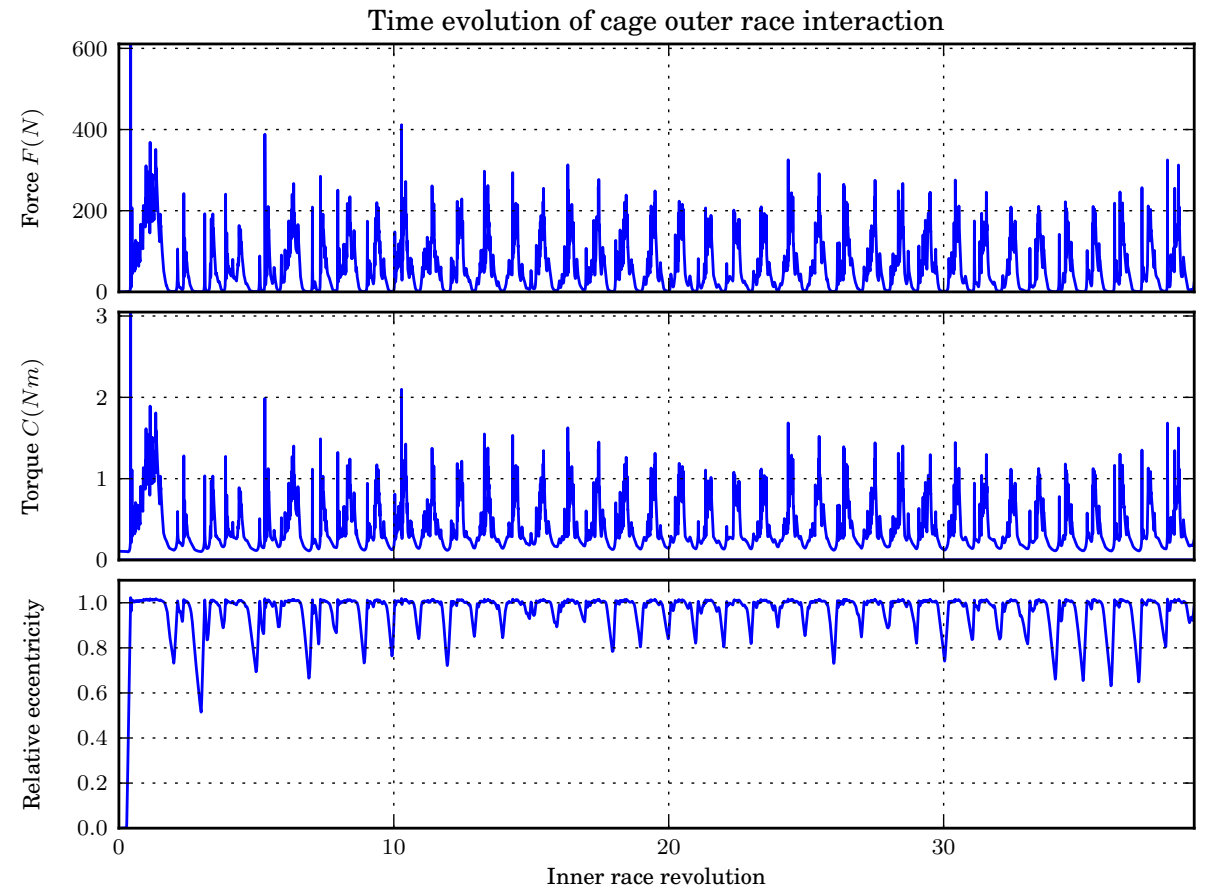

Figure 19: Evolution over time of conformal contact data between cage and outer race, case (b) 
With regard to cage interactions, contact loads at the guide lands are severe (see FIG.19). The huge peak of $600 \mathrm{~N}$ may be explained by the approximate initial values of the cage configuration. The cage is heavily squeezed onto the guide lands. In comparison with the previous case, here the contact is of greater magnitude and frequency. This results in high wear on the cage and premature

420 failure. Impacts on cage pockets are shown in FIG 20(a) Again, impacts are much more frequent, with a magnitude of the impacts greater than in the first load case. Small pocket clearances may explain the fact that most of the impacts have a value less than $200 N$. Some long duration contacts of small amplitude are also present. In Fig 20(b), it should be noted that cage impacts occur in a well defined area. Indeed, impacts occur in two orbital regions $\left[115^{\circ}, 180^{\circ}\right]$ and $\left[0^{\circ},-45^{\circ}\right]$ roughly. In the first region, ball-to-pocket contact force and ball velocity are in the same direction, and hence the balls seem to lead the cage in this area. In the second region, the cage is slowed down by the balls. These two simultaneous actions cause the cage to be crushed in a fairly constant direction; this is a direct consequence of the excessive ball circumferential excursion.
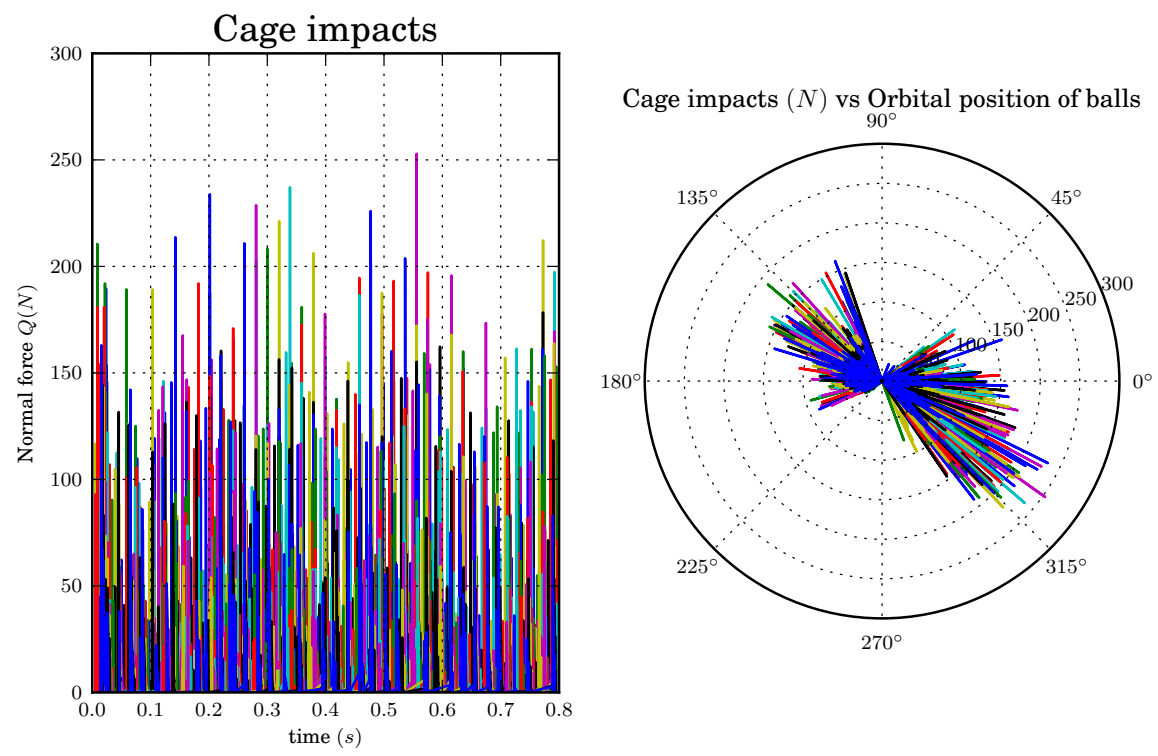

(a) Evolution over time of impacts on the (b) Impacts plotted as a function of the orcage bital position of the balls

Figure 20: Impacts on the cage, case (b)

The second load case corresponds to the experimental conditions experienced on a test rig. As expected, premature cage failure occurred during this test. After disassembly of the bearing, the outer race was burnt in quite a well defined orbital region. Good agreement is found when comparing the contact area predicted in FIG 17(a) with that observed in FIG 21(a). There are also burn 
marks all around the cage (see Fig 21(b)). A slight asymmetry in the burn marks is observed between the cage sides. This asymmetry may be explained by the non-centred axial position. Indeed, the cage axial position varies around $0.2 \mathrm{~mm}$ (see FIG 18(b)].

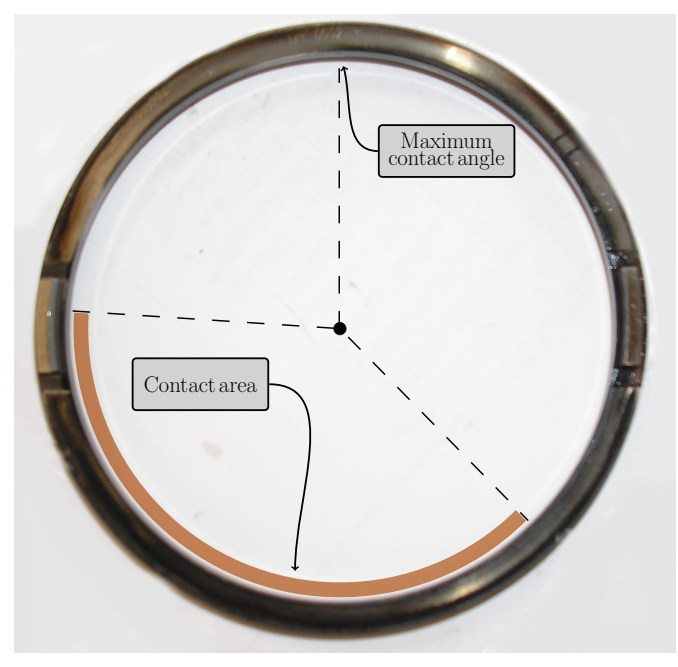

(a) Outer race

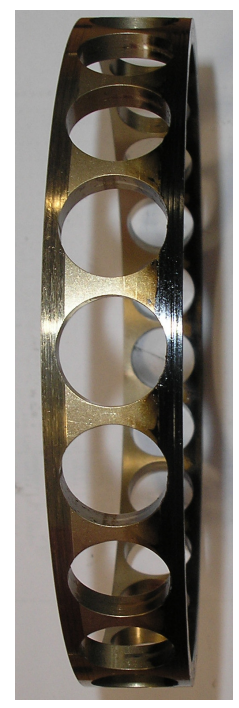

(b) Cage

Figure 21: Contact area on ball bearing components

Finally, owing to the high value of moment load, the ball bearing exhibits an obvious dysfunction. The premature failure was accurately predicted by the dynamic numerical analysis and confirmed by experimental observations. From the author's experience, without the hybrid short journal, the discontinuity in the contact force model leads to an absurd cage trajectory. On the other hand,

${ }_{445}$ if the viscoelastic hinge joint that constrains the inner race is removed, the cage is still constantly crushed against the outer race but the contact area moves all around the outer race. Thus, the viscoelastic hinge joint and the hybrid short journal model appear essential in managing the dynamic analysis of a ball bearing under such severe load conditions. 


\section{Acknowledgement}

The authors would like to thank the Airbus Helicopters Company for permission to publish this work.

[1] L. Zamponi, E. Mermoz, J.-M. Linares, J.-M. Sprauel, Impact of geometrical defects on bearing assemblies with integrated raceways in aeronautical gearboxes, Mechanism and Machine. Theory 44 (6) (2009) 1108-1120. doi:10.1016/j.mechmachtheory.2008.10.005. 
[2] A. B. Jones, A General Theory for Elastically Constrained Ball and Radial Roller Bearings Under Arbitrary Load and Speed Conditions, Journal of Fluids Engineering 82 (1960) 309-320. doi:10.1115/1.3662587.

[3] T. A. Harris, M. N. Kotzalas, Rolling bearing analysis, J Wiley, New York, 2001.

[4] C. Walters, The dynamics of ball bearings, Journal of Lubrication Technology 93 (1) (1971) 1-10. doi:10.1115/1.3451516.

[5] J. Kannel, S. Bupara, A simplified model of cage motion in angular contact bearings operating in the EHD lubrication regime, Journal of Tribology 100 (1978) 395-403. doi:10.1115/1.3453196

[6] C. Meeks, L. Tran, Ball bearing dynamic analysis using computer methods-part I: analysis, Journal of Tribology 118 (1996) 52-58. doi: $10.1115 / 1.2837092$

[7] P. K. Gupta, Advanced dynamics of rolling elements, Springer-Verlag, 1984.

[8] P. K. Gupta, On the Geometrical Imperfections in Ball Bearings, Journal of Tribology 110 (1988) 19-25. doi:10.1115/1.3261567.

[9] N. Tandon, A. Choudhury, An analytical model for the prediction of the vibration response of rolling element bearings due to a localized defect, Journal of Sound and Vibration 205 (3) (1997) 275-292. doi:http://dx. doi.org/10.1006/jsvi.1997.1031

[10] S. P. Harsha, Nonlinear dynamic response of a balanced rotor supported by rolling element bearings due to radial internal clearance ef515 ـ fect, Mechanism and Machine Theory 41 (2006) 688-706. doi:10.1016/ j.mechmachtheory.2005.09.003.

[11] S. P. Harsha, Nonlinear dynamic analysis of rolling element bearings due to cage run-out and number of balls, Journal of Sound and Vibration 289 (1-2) (2006) 360-381. doi:10.1016/j.jsv.2005.02.021.

${ }_{520}[12]$ C. Nataraj, S. P. Harsha, The effect of bearing cage run-out on the nonlinear dynamics of a rotating shaft, Communications in Nonlinear Science

a and Numerical Simulation 13 (4) (2008) 822-838. doi:10.1016/j.cnsns. 2006.07 .010

[13] S. P. Harsha, K. Sandeep, R. Prakash, Non-linear dynamic behaviors of rolling element bearings due to surface waviness, Journal of Sound and Vibration 272 (3-5) (2004) 557-580. doi:10.1016/S0022-460X (03)00384-5

[14] B. Changqing, X. Qingyu, Dynamic model of ball bearings with internal clearance and waviness, Journal of Sound and Vibration 294 (1-2) (2006) 23-48. doi:10.1016/j.jsv.2005.10.005. 
[15] A. Leblanc, D. Nelias, C. Defaye, Nonlinear dynamic analysis of cylindrical roller bearing with flexible rings, Journal of Sound and Vibration 325 (1-2) (2009) 145-160. doi:10.1016/j.jsv.2009.03.013

[16] T. Yao, Y. Chi, Y. Huang, Research on Flexibility of Bearing Rings for Multibody Contact Dynamics of Rolling Bearings, Procedia Eng. 31 (2011) (2012) 586-594. doi:10.1016/j .proeng.2012.01.1071.

[17] A. Lioulios, I. Antoniadis, Effect of rotational speed fluctuations on the dynamic behaviour of rolling element bearings with radial clearances, Int.

I. J. Mech. Sci. 48 (8) (2006) 809-829. doi:10.1016/j.ijmecsci.2006.03. 006.

[18] A. Shabana, Dynamics of Multibody Systems, Cambridge University Press, 2005.

[19] M. Géradin, A. Cardona, Flexible multibody dynamics: a finite element approach, John Wiley, 2001.

[20] P. Flores, J. Ambrósio, J. P. Claro, Dynamic Analysis for Planar Multibody Mechanical Systems with Lubricated Joints, Multibody System Dynamics 12 (1) (2004) 47-74. doi:10.1023/B:MUBO.0000042901.74498.3a.

[21] P. Flores, H. M. Lankarani, Spatial rigid-multibody systems with lubricated spherical clearance joints: modeling and simulation, Nonlinear Dynamics 60 (1-2) (2009) 99-114. doi:10.1007/s11071-009-9583-z.

[22] P. Flores, J. Ambrósio, J. Claro, H. Lankarani, C. Koshy, Lubricated revolute joints in rigid multibody systems, Nonlinear Dynamics 56 (3) (2009) 277-295. doi:10.1007/s11071-008-9399-2.

[23] Q. Tian, Y. Zhang, L. Chen, J. J. Yang, Simulation of planar flexible multibody systems with clearance and lubricated revolute joints, Nonlinear Dynamics 60 (4) (2009) 489-511. doi:10.1007/s11071-009-9610-0.

[24] Q. Tian, Y. Zhang, L. Chen, P. Flores, Dynamics of spatial flexible multibody systems with clearance and lubricated spherical joints, Computers \&

1. Structures 87 (13-14) (2009) 913-929. doi:10.1016/j.compstruc.2009. 03.006

[25] L. Xu, Y. Yang, Y. Li, C. Li, S. Wang, Modeling and analysis of planar multibody systems containing deep groove ball bearing with clear-

घ ance, Mechanism and Machine Theory 56 (2012) 69-88. doi:10.1016/ j.mechmachtheory.2012.05.009.

[26] L.-x. Xu, Y.-g. Li, An approach for calculating the dynamic load of deep groove ball bearing joints in planar multibody systems, Nonlinear Dynamics 70 (3) (2012) 2145-2161. doi:10.1007/s11071-012-0606-9. 
[27] Z. Qi, G. Wang, Z. Zhang, Contact analysis of deep groove ball bearings in multibody systems, Multibody System Dynamics.doi:10.1007/ s11044-014-9412-0.

[29] L. E. Stacke, D. Fritzson, P. Nordling, BEAST - a rolling bearing simulation tool, Proceedings of the Institution of Mechanical Engineers, Part K: Journal of Multi-body Dynamics 213 (2) (1999) 63-71. doi:10.1243/ 1464419991544063 .

[30] I. Nakhimovski, Contributions to the Modeling and Simulation of Mechanical Systems with Detailed Contact Analyses, Ph.D. thesis, Linkpings university (2006).

[31] C. Bovet, J.-M. Linares, L. Zamponi, E. Mermoz, Multibody modeling of non-planar ball bearings, Mechanics \& Industry. 14 (5) (2013) 335-345. doi:10.1051/meca/2013075

[32] G. Nijenbanning, C. H. Venner, H. Moes, Film thickness in elastohydrodynamically lubricated elliptic contacts, Wear 176 (1994) 217-229. doi:http://dx.doi.org/10.1016/0043-1648(94)90150-3.

[33] C. H. Venner, Multilevel solution of the EHL line and point contact problems, Ph.D. thesis, University of Twente (1991).

[34] B. Jacod, Friction in elasto-hydrodynamic lubrication, Ph.D. thesis, University of Twente (2002).

[35] L. Zamponi, E. Mermoz, J.-M. Linares, Etudes des méthodes de calcul des pressions de contact dans les roulements à pistes intégrées des boîtes de transmission aéronautiques, Mécanique \& Industries 8 (2007) 567-575. doi:10.1051/meca:2007081

[36] O. Bauchau, L. Trainelli, The vectorial parameterization of rotation, Nonlinear Dynamics 32 (1) (2003) 71-92. doi:10.1023/A:1024265401576.

[37] M. Géradin, A. Cardona, Kinematics and dynamics of rigid and flexible mechanisms using finite elements and quaternion algebra, Computational Mechanics 4 (1989) 115-135. doi:10.1007/BF00282414

[38] H. M. Hilber, T. Hughes, R. L. Taylor, Improved numerical dissipation for time integration algorithms in structural dynamics, Earthquake Engi4290050306.

[39] O. Bauchau, On the Modeling of Friction and Rolling in Flexible MultiBody Systems, Multibody System Dynamics 3 (3) (1999) 209-239. doi: 
[40] K. Johnson, Contact Mechanics, Cambridge University Press, 1987.

[41] S. Yasutomi, S. Bair, W. Winer, An application of a free volume model to lubricant rheology I - dependence of viscosity on temperature and pressure, Journal of Tribology 106 (1984) 291-302. doi:10.1115/1.3260907.

[42] O. Pinkus, B. Sternlicht, Theory of hydrodynamic lubrication by Oscar Pinkus and Beno Sternlicht, New York : McGraw-Hill, 1961.

[43] J. Frêne, D. Nicolas, B. Degueurce, D. Berthe, M. Godet, Hydrodynamic lubrication: bearings and thrust bearings, Vol. 33, Elsevier, 1997.

[44] P. K. Gupta, Dynamic loads and cage wear in high-speed rolling bearings, Wear 147 (1) (1991) 119-134. doi:10.1016/0043-1648(91)90123-C. 